Mon. Not. R. Astron. Soc. 000, 田 16 (2001) Printed 24 October $2018 \quad$ (MN LATEX style file v2.2)

\title{
M82-F: A Doomed Super Star Cluster?^
}

\author{
Linda J. Smith ${ }^{1}$ and John S. Gallagher, III $^{2}$ \\ ${ }^{1}$ Department of Physics and Astronomy, University College London, Gower Street, London, WC1E 6BT \\ ${ }^{2}$ Department of Astronomy, University of Wisconsin-Madison, 5534 Sterling, 475 North Charter St., Madison WI 53706, USA
}

Accepted 2001 April 23.

\begin{abstract}
We present high dispersion echelle spectroscopy of the very luminous, young super star cluster (SSC) 'F' in M82, obtained with the 4.2-m William Herschel Telescope (WHT), for the purpose of deriving its dynamical mass and assessing whether it will survive to become an old globular cluster. In addition to the stellar lines, the spectrum contains complex $\mathrm{Na}$ I absorption and broad emission lines from the ionized gas. We measure a stellar velocity dispersion of $13.4 \pm 0.7 \mathrm{~km} \mathrm{~s}^{-1}$, a projected half-light radius of $2.8 \pm 0.3$ pc from archival HST/WFPC2 images, and derive a dynamical mass of $1.2 \pm 0.1 \times 10^{6} \mathrm{M}_{\odot}$, demonstrating that M82-F is a very massive, compact cluster. We determine that the current luminosity-to-mass ratio $\left(L_{V} / M\right)_{\odot}$ for M82-F is $45 \pm 13$. Comparison with spectral synthesis models shows that $\left(L_{V} / M\right)_{\odot}$ is a factor of $\sim 5$ higher than that predicted for a standard Kroupa (2001) initial mass function (IMF) at the well-determined age for M82-F of $60 \pm 20 \mathrm{Myr}$. This high value of $\left(L_{V} / M\right)_{\odot}$ indicates a deficit of low mass stars in M82-F; the current mass function (MF) evidently is 'top-heavy'. We find that a lower mass cutoff of $2-3 \mathrm{M}_{\odot}$ is required to match the observations for a MF with a slope $\alpha=2.3$. Since the cluster apparently lacks longlived low mass stars, it will not become an old globular cluster, but probably will dissolve at an age of $\leqslant 2 \mathrm{Gyr}$. We also derive up-dated luminosity-to-mass ratios for the younger SSCs NGC 1569A and NGC 1705-1. For the first object, the observations are consistent with a slightly steeper MF $(\alpha=2.5)$ whereas for NGC 1705-1, the observed ratio requires the MF to be truncated near $2 \mathrm{M}_{\odot}$ for a slope of $\alpha=2.3$. We discuss the implications of our findings in the context of large scale IMF variations; with the present data the top-heavy MF could reflect a local mass segregation effect during the birth of the cluster. M82-F likely formed in a dense molecular cloud; however, its high radial velocity with respect to the centre of M82 $\left(\sim-175 \mathrm{~km} \mathrm{~s}^{-1}\right)$ suggests it is on an eccentric orbit and now far from its birthplace, so the environment of its formation is unknown.
\end{abstract}

Key words: galaxies: evolution - galaxies: individual (M82) - galaxies: starburst galaxies: star clusters - galaxies: stellar content

\section{INTRODUCTION}

M82 is usually considered the prototype of starburst galaxies since the luminosity and star formation rate of its central star-forming region is comparable to the luminosity of a typical giant spiral galaxy (e.g. Telesco 1988; Lester et al. 1990). Imaging studies at optical and infrared wavelengths have shown that young, compact, luminous star clusters ('super

\footnotetext{
* Based in part on observations with the NASA/ESA Hubble Space Telescope, obtained at the Space Telescope Science Institute, which is operated by the Association of Universities for Research in Astronomy (AURA), Inc., under NASA contract NAS 5-26555. Also based on observations obtained with the William Herschel and the WIYN telescopes.
}

star clusters' or SSCs) are very numerous in the actively star-forming regions of M82 (O'Connell \& Mangano 1978; O'Connell et al. 1995; Satyapal et al. 1995, 1997; de Grijs et al. 2001). M82 provides an excellent and nearby setting in which to study the properties of young SSCs. We adopt a distance to M82 of $3.6 \mathrm{Mpc}$ by assuming that it is at the same distance as M81 (Freedman et al. 1994), consistent with the red giant branch tip distance to M82 found by Sakai \& Madore (1999).

The presence of luminous, massive SSCs in starburst galaxies leads to the question: are these really 'young globular star clusters' (e.g. Schweizer \& Seitzer 1993; Whitmore et al. 1993; O'Connell, Gallagher \& Hunter 1994; Meurer et al. 1995)? Differences in cluster luminosity functions between those for young SSCs in starbursts and those of ancient 
globular cluster systems may present a problem. While globular star cluster luminosity functions peak at $M_{V} \approx-7.5$ (corresponding to $M \sim 10^{5} \mathrm{M}_{\odot}$; see Harris 1991; Ashman, Conti \& Zepf 1995), the raw luminosity functions of clusters spawned in starbursts typically resemble the power law distributions found for open star clusters (e.g. Whitmore et al. 1999; Zhang \& Fall 1999), although incompleteness may be an issue (de Grijs, O'Connell \& Gallagher 2001). A further complication can arise in the evolution of populations of star clusters within galaxies. Fritze - v. Alvensleben (1998, 1999) emphasized the possible importance of differential survival rates, which favour dense, massive star clusters, such as compact SSCs, and might transform the power luminosity functions of star clusters at birth into those of globular clusters (see also Chernoff \& Weinberg 1990; Elmegreen \& Efremov 1997; Gnedin \& Ostriker 1997). Luminosity function measurements alone for populations of star clusters do not resolve this issue.

The questions of masses and densities of SSCs are therefore critical; do SSCs have the mass densities of globular star clusters? This is a necessary but not a sufficient condition for SSCs to survive for $\sim 10$ Gyr time scales to become globular clusters. Ho \& Filippenko (1996a,b) measured stellar velocity dispersions for two SSCs, NGC 1569A and NGC 1705-1, with the HIRES echelle spectrograph on the Keck I 10-m telescope. Combining these data with half-light radii in the literature derived from observations with the Hubble Space Telescope (HST), they found dynamical masses consistent with those of globular clusters. Both of these SSCs are relatively bright ( $V=14.8$ and 14.7 , respectively), young (ages of $<20 \mathrm{Myr}$ ), and in small galaxies with low metallicities.

The recognition that compact SSCs may indeed be proto-globular clusters opens up the possibility of studying their formation conditions, evolution and destruction processes. One issue of particular interest is whether SSCs have sufficient numbers of low mass stars to remain as bound systems over long time-scales. The slope of the stellar initial mass function (IMF) is critical since too high a fraction of high mass stars will cause the cluster to evaporate within a few Gyr due to stellar mass-loss (e.g. Chernoff \& Weinberg 1990; Goodwin 1997; Takahashi \& Portegies Zwart 2000). It is not obvious whether all young SSCs have 'normal' MFs. For example, the best studied Galactic example, NGC 3603, has a normal MF extending down to $<1 \mathrm{M}_{\odot}$ according to Eisenhauer et al. (1998). The compact young Galactic centre clusters, the Arches and Quintuplet, appear to have remarkably flat MFs for $\mathrm{M}>10 \mathrm{M}_{\odot}$ (Figer et al. 1999), although they have probably undergone rapid mass segregation (Kim, Morris \& Lee 1999) and could even represent the dense cores of dissolved clusters (Gerhard 2001).

In the LMC SSC R136, Massey and Hunter (1998) demonstrated a normal mass function for stars with $M \geqslant$ $2.8 \mathrm{M}_{\odot}$ while Sirianni et al. (2000) find that the mass function flattens below $2 \mathrm{M}_{\odot}$; the $\mathrm{R} 136 \mathrm{MF}$ could be slightly

1 There is some confusion in the literature over the use of the term 'IMF' to describe the observed properties of evolved star clusters. We will use the term ' $\mathrm{IMF}$ ' for the theoretical initial distribution of masses, and the term 'mass function (MF)' to refer to the present-day mass distribution of a cluster inferred from observation. 'top-heavy' compared to that for LMC field stars. Similarly, Sternberg (1998) suggests a deficiency of low mass stars in NGC 1705-1. Spectra of SSCs in the peculiar galaxy NGC 1275 taken with the Keck I telescope show unusually strong H-Balmer lines; Brodie et al. (1998) interpret these as evidence for flat MFs in clusters that are about $350 \mathrm{Myr}$ old.

More generally there has been much debate over the slope of the IMF in starburst galaxies, and particularly, in M82. Rieke et al. (1980) first suggested that the M82 starburst has a deficit of stars below $3 \mathrm{M}_{\odot}$ on the basis of its high $K$ band luminosity and relatively small dynamical mass. Rieke et al. (1993) used up-dated models and observational constraints from McLeod et al. (1993) to demonstrate further that the M82 starburst has an MF biased towards more massive stars. Doane \& Mathews (1993) also find that the M82 MF must be top-heavy to explain both the high supernova rate and low dynamical mass. On the other hand, Satyapal et al. $(1995,1997)$ derived a smaller value for the extinction-corrected $K$ band luminosity and found that the MF could be consistent with a Salpeter slope.

In a previous paper (Gallagher \& Smith 1999; hereafter Paper I), we presented a spectroscopic study of two M82 SSCs, denoted F and L by O'Connell \& Mangano (1978). M82-F is one of the most luminous SSCs known (O'Connell et al. 1995) and is located $440 \mathrm{pc}$ south-west of the nucleus. We found that its radial velocity indicated that it could be buried deep within M82 and fortuitously viewed through a hole in the dust layer. Conversely, the nearby cluster M82$\mathrm{L}$ is highly reddened but its radial velocity indicates that it lies near the middle of the M82 disc. Our blue spectrum of M82-F shows mid-B type spectral features and the red spectra of M82-F and M82-L have a strong CaII triplet and numerous F- and G-type absorption lines. We compared the blue spectrum of M82-F with theoretical model cluster spectra using the PEGASE spectral synthesis code (Fioc \& Rocca-Volmerange 1997), and derived an age of $60 \pm 20 \mathrm{Myr}$. For M82-L, we find that the similarities in the strength of the Ca II triplet and overall spectral appearance with M82-F suggest a similar age.

The location away from the central starburst and compactness of M82-F make it an ideal candidate in M82 for determining its dynamical mass by measuring its size and internal velocity dispersion. It is an important addition to SSCs with dynamical masses because it is older and probably more metal-rich than the two SSC dynamical mass measurements by Ho \& Filippenko (1996a,b), but has the disadvantage of being relatively faint, $V=16$. In this paper we present the results of such a study based on high dispersion echelle spectroscopy obtained at the 4.2-m William Herschel Telescope (WHT). We compare the dynamical mass of M82F with those determined for other young SSCs by Ho and Filippenko (1996a,b), using up-dated parameters. In particular, we discuss its luminosity-to-mass ratio in the context of IMF models that lack lower mass stars. The form of the IMF in turn affects the ability of the cluster to survive as a bound system and thus its status as a young globular star cluster. We also discuss the dynamics of neutral and ionized gas in the line of sight to M82-F through the analysis of the $\mathrm{Na}$ I interstellar lines and various H II region emission lines. 


\section{OBSERVATIONS AND DATA REDUCTION}

\section{$2.1 \quad$ Images}

In Fig. 1, the locations of clusters F and L within M82 are shown using $I$-band images taken with the WIYN $3.5-\mathrm{m}$ telescope and the Wide Field Planetary Camera (WFPC2) onboard HST. The WFPC2 image was obtained from the $H S T$ archive and is an average of two $200 \mathrm{~s}$ exposures in the F $814 \mathrm{~W}$ filter. There are also $V$-band (two $350 \mathrm{~s}$ exposures in the F555W filter) and $B$-band (two $400 \mathrm{~s}$ exposures in the F439W filter) images available. These images were taken as part of the GO 7446 program (R. W. O'Connell, P.I.) to investigate star clusters and other features of the visible surface of the M82 starburst (see de Grijs et al. 2000, 2001). They were not designed for measurements of bright star clusters such as M82-F, and this introduced some complications.

Images of M82-F and M82-L are both on the WFPC2 WF4 CCD, which has an image scale of 0.1 arcsec pixel $^{-1}$. We worked from combined pairs of images in each filter, and used standard IRAF software to remove cosmic rays (there were no major cosmic ray hits on or near M82-F). The F439W short exposure images are unsaturated and give a true measure of the M82-F brightness profile. Both the F555W and F814W images contain 9 saturated pixels (due to the WFPC2 A/D converter response) in the M82-F cluster centre. We corrected these by using the F439W image as a template and replaced the saturated pixels with values scaled from the surrounding regions of the cluster in the F555W and F814W bands. To estimate the error, we arbitrarily increased the values of all saturated pixels by 30 per cent, and find that this leads to an increase of slightly more than 0.1 magnitudes in the brightness of M82-F in both the F555W and F814W images. The method we have used assumes that there is no radial colour gradient present in M82-F. Any colour changes are expected to be small since typical gradients of at most $\sim 0.1$ mag are observed in rich LMC clusters (Elson, Fall \& Freeman 1987). We therefore adopt an error of \pm 0.10 mag for the $V$ and $I$ magnitudes of M82-F. The F439W and F814W images of M82-F are shown in Fig. 2. M82-L is very bright in the F814W-band but essentially absent in F439W. We also see that M82-F has a companion star cluster at a projected distance of 0.7 arcsec along a position angle of $50^{\circ}$.

Aperture photometry was performed on M82-F and its companion. The region of the companion cluster was replaced with the mean background for the M82-F photometry. A circular aperture with a 7 pixel radius was chosen for the total magnitude measurement. The background was measured from the centroid of the distribution of pixel brightnesses in an annulus at 12-14 pixels. Magnitudes were transformed to the Landolt $B V I$ system following the precepts of Holtzman et al. (1995). We obtain $V=16.12$ and $V-I=1.51 \pm 0.10$ (Table 1 ). Using a smaller aperture of

2 The Image Reduction and Analysis Facility (IRAF) is distributed by the National Optical Astronomy Observatories which is operated by the Association of Universities for Research in Astronomy, Inc. under cooperative agreement with the National Science Foundation. STSDAS is the Space Telescope Science Data Analysis System; its tasks are complementary to those in IRAF. radius 0.5 arcsec, we obtain $V=16.31 \pm 0.10$; O'Connell et al. (1995) used observations taken with the original WFPC to estimate a deconvolved $V=16.3$ within the same radius.

Photometry of the companion star cluster was performed by estimating a constant background which was derived by assuming that M82-F has a symmetric structure, and then using the mean count rates on the side opposite of the cluster to the companion. This procedure yielded for the M82-F companion cluster $V=18.4 \pm 0.2$ and $B-V=0.9 \pm 0.3$ using a 5 pixel radius circular aperture. Due to the faintness of this object with respect to the galaxy background and proximity of M82-F, we could not obtain reliable photometry from the F814W WFPC2 image.

From circular aperture photometry on M82-F, we measure a half-light radius $r_{\mathrm{h}}(\mathrm{F} 439 \mathrm{~W})=0.22 \pm 0.02 \operatorname{arcsec}(1$ pixel $=0.1$ arcsec). The ratio of major to minor axes was determined to be about 1.3 using an ellipse fitting routine in the IRAF software package. This gives a negligible ellipticity correction of $<0.01$ arcsec for the effective radii of circular apertures used for the photometry. To correct for the effects of blurring of the point-spread-function (PSF) of the WF cameras of WFPC2, we used the simple approach of Phillips et al. (1997) who suggest subtracting $r_{\mathrm{h}}=0.15$ arcsec from the observed value in quadrature. This then gives a projected half-light radius of $r_{\mathrm{h}}=0.16 \operatorname{arcsec}$ or $2.8 \pm 0.3 \mathrm{pc}$ for an adopted distance to M82 of 3.6 Mpc. O'Connell et al. (1995) derived dimensions of $9 \times 5 \mathrm{pc}$ or a mean FWHM of $2.8 \mathrm{pc}$ from their deconvolved WFPC images.

The SSC NGC 1705-1 is an important comparison object for M82-F. Short exposure, unsaturated archival WFPC2 images of NGC 1705-1 on the PC are available in the F380W and F439W filters from HST program GO 7506 (M. Tosi, P.I.). The guiding in these exposures is not good. Images are elongated in both exposures, with the problem being more serious in the F439W than F380W image. These images show an extended halo of luminous stars around NGC 1705-1 and a bright object nearby that is identified as the second brightest cluster in the galaxy by O'Connell et al. (1994).

The half-light radius of NGC 1705-1 is uncertain, with O'Connell et al. (1994) estimating 0.14 arcsec and Meurer et al. (1995) re-deriving 0.04 arcsec from the same WFPC PC data. We therefore measured $r_{\mathrm{h}}$ from the F380W archival WFPC2 image which appears to have the best image quality. Our technique is the same as that used for M82-F. A PSF correction was derived from measurements of stars surrounding the cluster to be $1.8 \mathrm{WFPC} 2 / \mathrm{PC}$ pixels, or 0.082 arcsec. The measured projected half-light radius for the central star cluster measured from multiple aperture photometry is 2.25 pixels, and thus $r_{\mathrm{h}}=0.062 \pm 0.01$ arcsec. O'Connell et al. (1994) measured a distance to NGC 1705 of $5.0 \pm 2.0 \mathrm{Mpc}$; this has been revised recently by Tosi et al. (2001, in prep.) to $5.3 \pm 0.8 \mathrm{Mpc}$ from the tip of the red giant branch. We adopt this new distance and find that $r_{\mathrm{h}}=1.6 \pm 0.4 \mathrm{pc}$.

We also re-measured the total magnitude of NGC 17051 from aperture photometry of the 140 s exposure F439W image. The photometry is complicated by the presence of the nearby bright cluster (at a projected distance of 0.9 arcsec) and the extended halo. Aperture photometry with a radius of 25 pixels ( $=1.2$ arcsec), a reasonable estimate of the maximum cluster size, gives $m_{\mathrm{F} 439 \mathrm{~W}}=14.85$ with small aperture photometry of the companion cluster subtracted. This SSC 

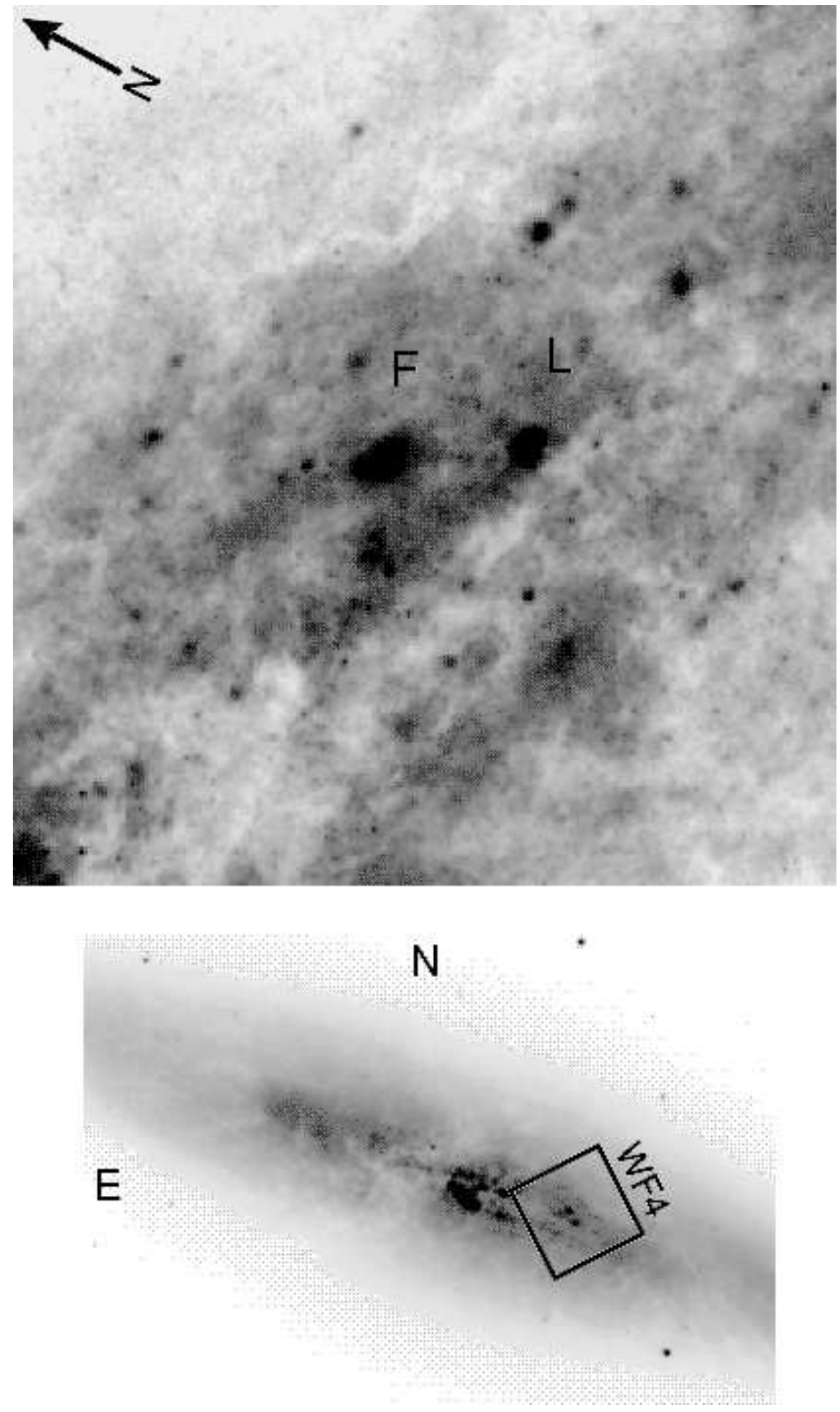

Figure 1. These $I$-band images show the locations of clusters F and L in M82. The lower picture was taken with a CCD camera on the WIYN $3.5-\mathrm{m}$ telescope in $\approx 1$ arcsec seeing. The location of the $25 \times 25$ arcsec section of the WFPC2 WF4 CCD containing the clusters is marked. The upper image is from the WFPC2 F814W observations, and displays the complex small scale structure of star clusters and dust lanes in the region of M82 where clusters F and L are located. 


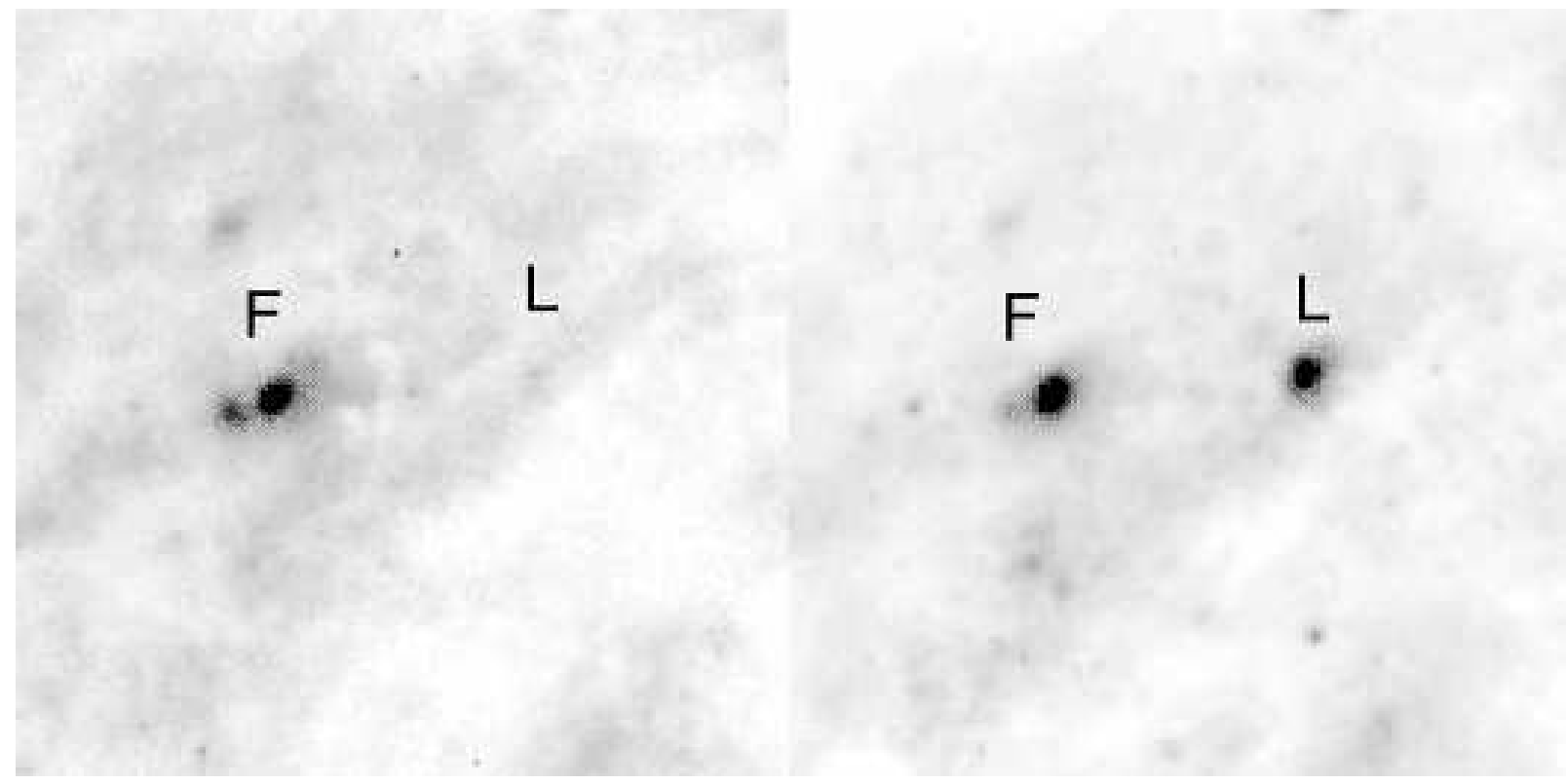

Figure 2. Blue (left panel, F439W filter) and red (right panel, F814W filter) WFPC2 HST observations of an 8 arcsec field around M82 clusters F and L are shown. M82-L is so heavily reddened as to be invisible in the blue, while the companion cluster located to the left and slightly below M82-F is most pronounced in the blue.

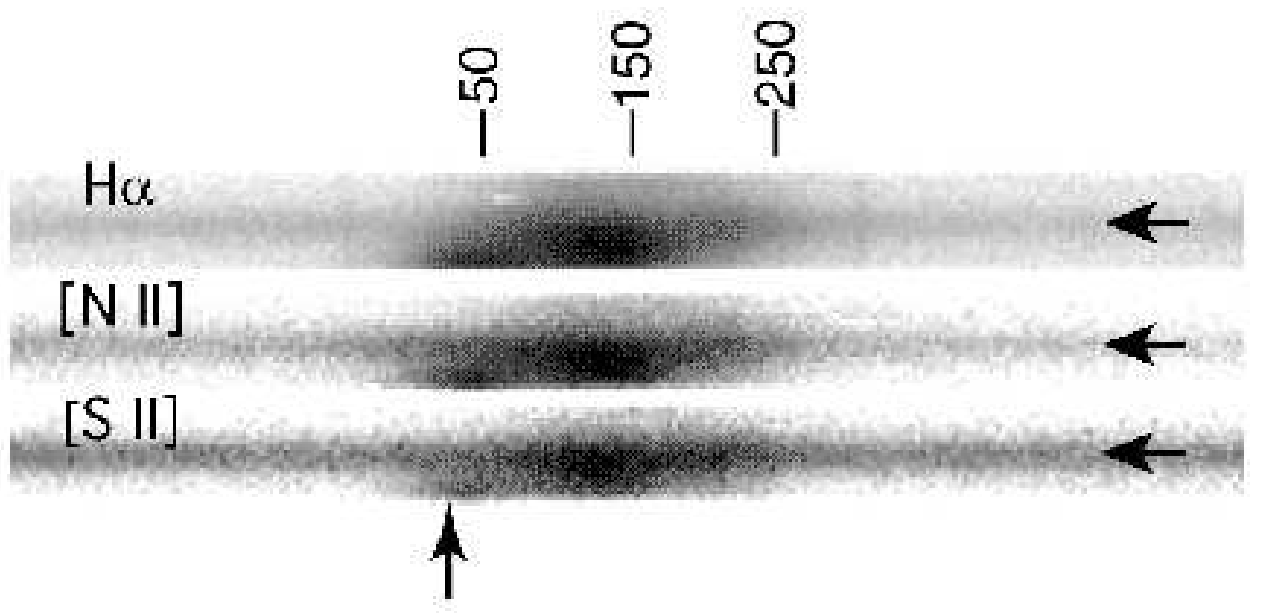

Figure 3. Greyscale images are shown for the individual orders of echelle spectra from the first night of WHT observations. The major emission lines are marked, where we have chosen the stronger of the [N II] and [S II] doublets. A heliocentric velocity scale is plotted above the spectra, and the location of M82-F along the 6 arcsec slit in each echelle spectral order and in radial velocity is marked with arrows.

has $B-V \approx 0$, in which case $m_{\mathrm{F} 439 \mathrm{~W}} \approx B=14.9 \pm 0.1$, where the error range includes a provision for transformation problems (see Holtzman et al. 1995). Aperture photometry to 50 pixels with the companion cluster subtracted gives $B=14.75 \pm 0.1$. This value agrees well with the value of $V=14.7$ reported by O'Connell et al. (1994) for NGC 1705-1 for a 3.4 arcsec radius aperture. We adopt $B=V=14.9 \pm 0.1$ for NGC 1705-1.

\subsection{Spectra}

M82-F was observed with the Utrecht echelle spectrograph (UES) at the Nasmyth focus of the WHT on 1999 February 26 and 27 . The 31.6 grooves $\mathrm{mm}^{-1}$ echelle grating was used with a $2048 \times 2048$ SITe CCD to record the wavelength range $5760-9140 \AA$ in 36 orders. The orders overlap by $34-$ $55 \AA$ since the size of the detector exceeds the free spectral range of this grating (59-143 $\AA$ ). M82-F was observed with a slit length of 6 arcsec (corresponding to 18 spatial pix- 
els) to ensure no overlap between adjacent orders while still providing a reasonable sample of the background. The slit was kept at a position angle of $139^{\circ}$ throughout the observations. A slit width of 1.3 arcsec was used which projected to 2.3 detector pixels on the detector (as measured from $\mathrm{ThAr}$ comparison arc lines), giving a resolving power of 45000 . The total integration time over the two nights was $23200 \mathrm{~s}$ in seeing conditions of $0.8-1$ arcsec. In addition, two atmospheric standards and eight template stars were observed ranging in spectral type from A7 III to M2 Iab.

The echelle data were reduced using the FIGARO software package (Shortridge et al. 1997). Each frame was cleaned of cosmic rays, bias corrected and divided by a normalized flat field. The individual orders were next extracted for the object and two adjacent background regions (sky + galaxy) and wavelength calibrated. The orders were then background-subtracted, binned to $4 \mathrm{~km} \mathrm{~s}^{-1}$ wide intervals, and co-added. Finally the orders were merged in the overlap regions and normalized to unity using spline fits. The final spectrum of $\mathrm{M} 82-\mathrm{F}$ has a resolution of $8 \mathrm{~km} \mathrm{~s}^{-1}$ and a signal-to-noise per pixel of $15-25$. The template spectra were reduced in exactly the same manner.

\section{INTERSTELLAR MEDIUM}

\subsection{Ionized Gas}

To investigate the dynamics of the ionized gas in the line of sight to M82-F, we simply took the median of the echellograms obtained on the first night since this night had the best seeing conditions. The resulting image is shown in Fig. 3 in the region of the $\mathrm{H} \alpha,[\mathrm{N} \mathrm{II}]$ and [S II] emission lines. All these emission lines show an identical dynamical structure. Examining the line profiles along the 6 arcsec slit (= 18 pixels), we find that there are at least three components present at $+50,+120$ and $+180 \mathrm{~km} \mathrm{~s}^{-1}$ with an overall FWHM of $\sim 120 \mathrm{~km} \mathrm{~s}^{-1}$. The peak of the $\mathrm{CO}$ rotation curve lies between the $120 \mathrm{~km} \mathrm{~s}^{-1}$ and $50 \mathrm{~km} \mathrm{~s}^{-1}$ emission components (Shen \& Lo 1995). The heliocentric radial velocity of M82-F is measured to be $+24 \mathrm{~km} \mathrm{~s}^{-1}$ from the Ca II triplet lines, in good agreement with the value of $+35 \mathrm{~km} \mathrm{~s}^{-1}$ obtained in Paper I.

The first two velocity components are resolved in the first three spatial pixels but appear to merge to produce two components at +124 and $+180 \mathrm{~km} \mathrm{~s}^{-1}$ at the position of M82-F on the slit (Fig. B). The strongest component at $+124 \mathrm{~km} \mathrm{~s}^{-1}$ then weakens and the last three spatial pixels show an unresolved broad $\left(\mathrm{FWHM}=135 \mathrm{~km} \mathrm{~s}^{-1}\right)$ emission feature at $+137 \mathrm{~km} \mathrm{~s}^{-1}$. In Paper I we found that the same emission lines are split into two components at +114 and $+181 \mathrm{~km} \mathrm{~s}^{-1}$ with the lower velocity component occurring along the sight line between M82-F and M82-L. The positions angles used in the two sets of observations are quite different: $139^{\circ}$ for the UES and $20^{\circ}$ for the observations in Paper I. In this region of M82 the CO emission extends over $\approx 150 \mathrm{~km} \mathrm{~s}^{-1}$ and shows a pronounced extension towards low radial velocities that also is seen in radio $\mathrm{H}$ recombination lines (e.g. Seaquist et al. 1996). We conclude that no association exists between ionized gas and M82-F; this is not surprising given the age and high orbital velocity of the cluster (see Sect. 7.3).
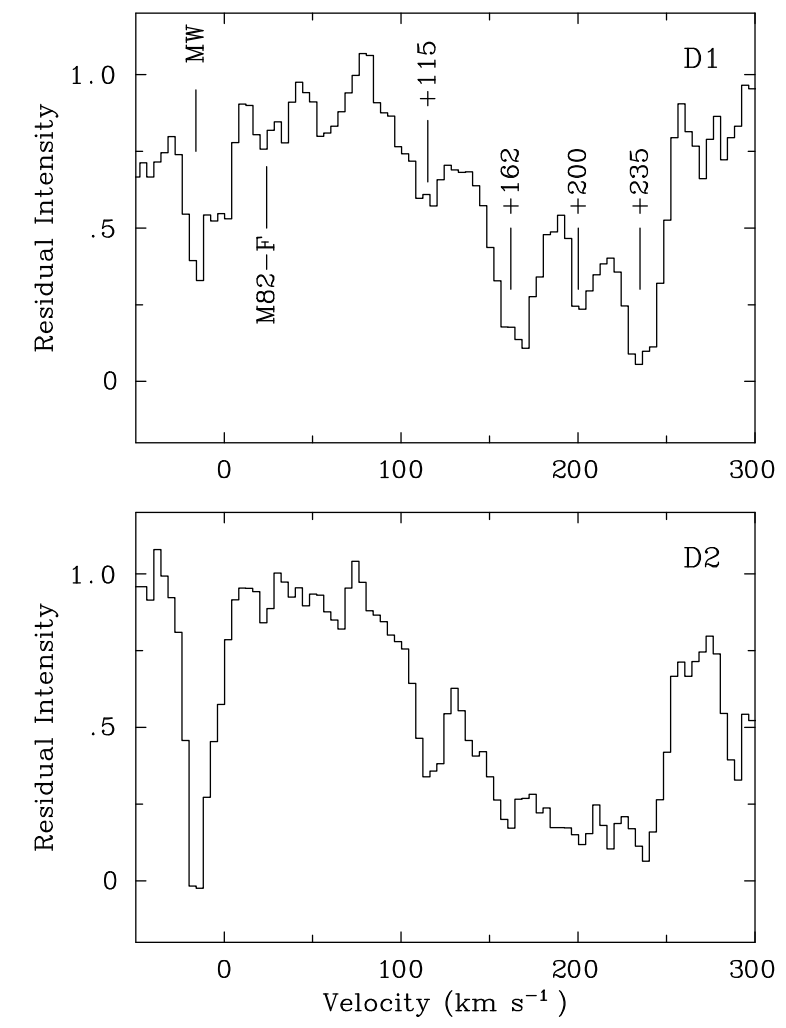

Figure 4. Portion of the WHT+UES spectrum of M82-F showing the region of the interstellar NaI D lines. The velocity scale is heliocentric and the spectra are normalized. The velocities of the resolved components are indicated. The strong narrow feature at $-16 \mathrm{~km} \mathrm{~s}^{-1}$ is due to interstellar absorption in the Milky Way. The radial velocity of M82-F is also indicated.

\subsection{Neutral Sodium Absorption}

Figure 4 shows the $\mathrm{NaI} \mathrm{D}_{1}$ and $\mathrm{D}_{2}$ absorption line profiles observed towards M82-F. The presence of multiple sharp components in the $\mathrm{D}_{1}$ line demonstrates that this feature is due to intervening interstellar gas rather than stellar photospheres as assumed in some previous studies (e.g. Saitō et al. 1984; Götz et al. 1990). The individual components can be seen more clearly in the $\mathrm{D}_{1}$ line, indicating that optical depth variations are occurring between the $\mathrm{D}_{1}$ and $\mathrm{D}_{2}$ lines; these features are formed in low column density neutral gas in M82. The heliocentric centroid velocity of about $190 \mathrm{~km} \mathrm{~s}^{-1}$ agrees with the $\mathrm{Na}$ I absorption radial velocities in Paper I.

With the resolution of the echelle, we see that these lines extend from approximately 115 to $240 \mathrm{~km} \mathrm{~s}^{-1}$ at a location in M82 where the rotation peak is blueshifted to $\sim 80 \mathrm{~km} \mathrm{~s}^{-1}$ (see Sect. 3.1). Adopting a systemic velocity of $200 \pm 10 \mathrm{~km} \mathrm{~s}^{-1}$ and a rotational velocity amplitude of $\approx 100 \mathrm{~km} \mathrm{~s}^{-1}$ for M82 (Yun, Ho \& Lo 1993; Achtermann \& Lacy 1995), we see that absorbing gas in circular orbits within the disc must lie in the approximate velocity range of $100 \mathrm{~km} \mathrm{~s}^{-1}<V_{c}<200 \mathrm{~km} \mathrm{~s}^{-1}$ i.e. between the rotation curve peak and zero velocity relative to the M82 system. Figure 4 shows this is where the bulk of the Na I absorption is located, although lines redshifted beyond the systemic ve- 


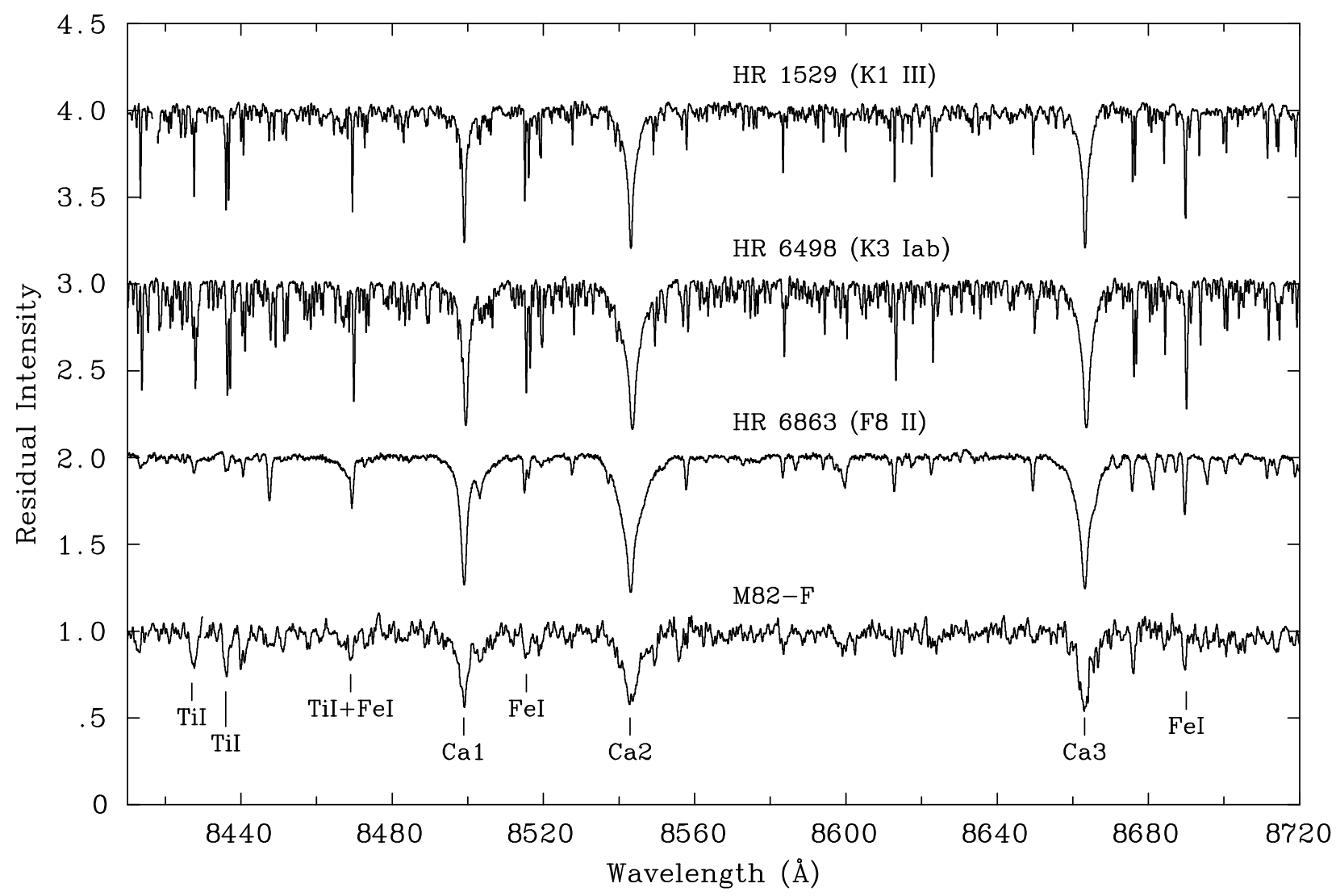

Figure 5. WHT +UES spectra covering the Ca II triplet region at a resolution of $8 \mathrm{~km} \mathrm{~s}^{-1}$ for M82-F and the three template stars which show the closest spectral match. The spectra have been normalized and shifted in velocity space to match the radial velocity of M82-F, and the M82-F spectrum has been lightly smoothed for presentation purposes. Absorption features due to the Ca II triplet and Fe I and Ti I are indicated.

locity are forbidden for circular rotation. This gas could be associated with infalling tidal debris or gas outflow from M82, although the H I map of Yun et al. (1993) suggests that most of the gas near M82-F has a velocity of less than $150 \mathrm{~km} \mathrm{~s}^{-1}$.

On the other hand, the CO 1-0 maps of Shen \& Lo (1995) and Sofue et al. (1992) show a large redward velocity extent to the south-west of the nucleus that becomes more pronounced above the galaxy's midplane. Since M82 is seen nearly edge on, the location of M82-F with respect to the mid-plane is uncertain, and we could be sampling some of this gas. Shen \& Lo (1995) propose that the high velocity dispersion molecular emission originates in spiral arms where previous starburst activity disrupted the interstellar medium. High angular resolution maps of gas kinematics in the outer disc of M82 would be very useful in disentangling the multiple interactions in this peculiar system.

\section{PARAMETERS OF M82-F}

The wavelength region of the UES observations (5760$9140 \AA$ ) was chosen because, as discussed by Ho \& Filippenko (1996a,b), and demonstrated by our intermediate dispersion spectra of M82-F (Paper I), the spectral region longward of $5000 \AA$ is dominated by the light of cool evolved stars. Thus by cross-correlating the cluster spectrum with a suitable template spectrum, it is possible to recover the lineof-sight velocity dispersion of the cluster, and hence derive its dynamical mass by application of the virial theorem.

\subsection{The Velocity Dispersion}

The spectrum of M82-F was compared with the spectra of the eight template stars to find the best match in spectral type. In Fig. 5, we show the region containing the CaII triplet for M82-F and the closest three template stars: HR 6863 (F8 II), HR 1529 (K1 III) and HR 6498 (K3 Iab). The metal lines in this wavelength region are mainly due to Fe I and Ti I. Comparison of the ratios of the strengths of these lines to the Ca II triplet lines shows that the closest spectral match is to the two $\mathrm{K}$ stars. We have used these three stars as templates and cross-correlated their spectra with that of M82-F. We chose four spectral regions which are free of

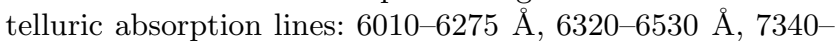
$7590 \AA$, and $7705-8132 \AA$ for the analysis. All these regions gave a strong cross-correlation signal; an example is shown in Fig. 6 where we also show the spectrum of an atmospheric standard. We ignored the region containing the Ca II triplet because these lines are saturated in the template spectra and broad Paschen absorption lines from early-type stars are present in the M82-F spectrum.

The resulting cross-correlation peaks were fitted with 

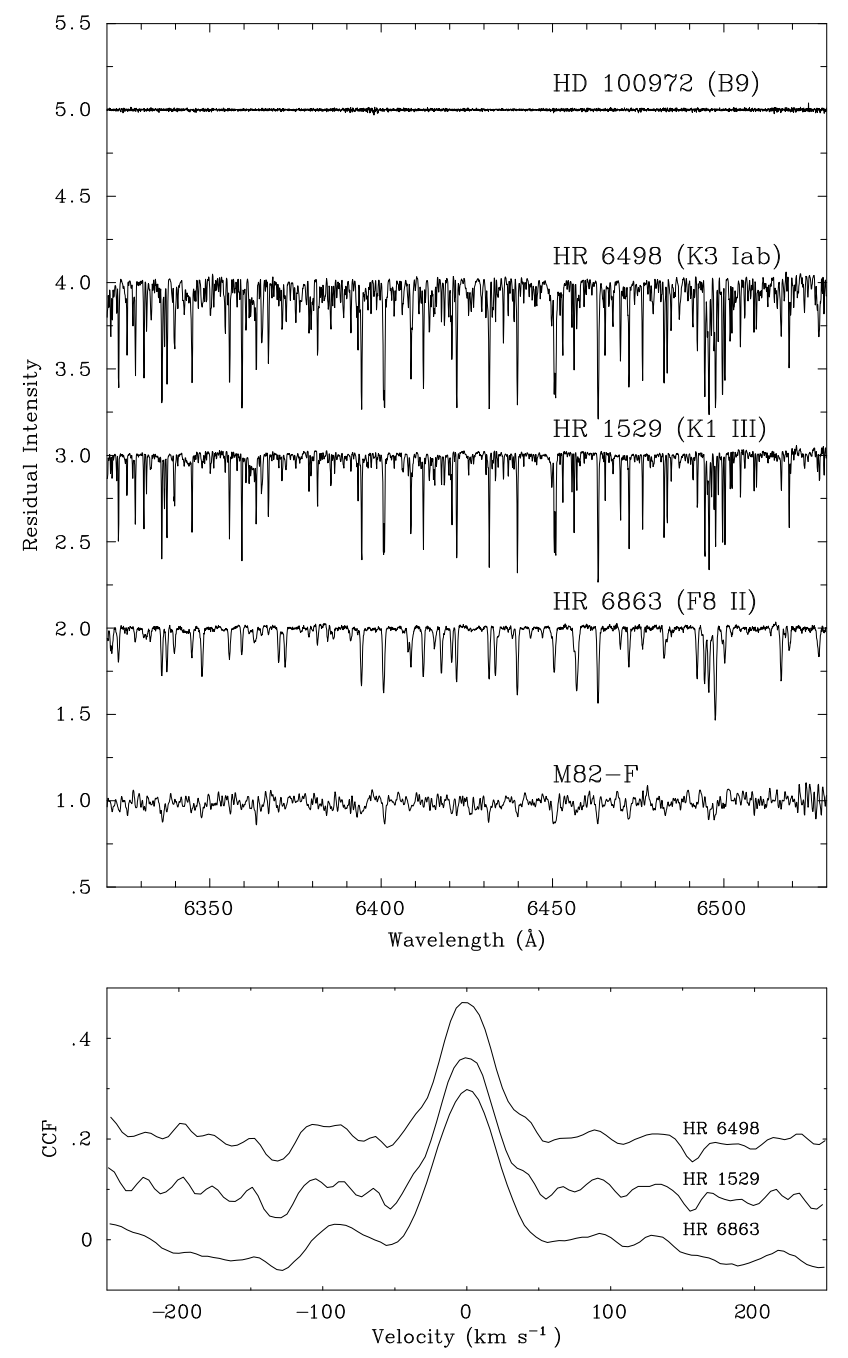

Figure 6. Top panel: Comparison of the normalized spectra of M82-F and the three template stars for one of the wavelength regions used for the cross-correlation. The spectrum of the B9 atmospheric standard is also shown to demonstrate the absence of telluric features. Again the spectra have been shifted to match the velocity of the cluster, and that of M82-F is lightly smoothed for presentation purposes. Bottom Panel: The cross-correlation functions (CCFs) between the cluster and the three template stars. The velocity dispersion of M82-F can be derived from the FWHM of the CCF.

Gaussian profiles using least-squares to obtain the FWHMs. The relationship between the FWHM and the velocity dispersion was empirically calibrated by broadening the template spectra with Gaussians of different velocity dispersions and cross-correlating with the original spectra. We find that relative to HR 6863 (F8 II), HR 1529 (K1 III) and HR 6498 (K3 Iab), the velocity dispersion of M82-F is $13.4 \pm 0.7$, $15.4 \pm 1.6$ and $15.3 \pm 1.5 \mathrm{~km} \mathrm{~s}^{-1}$ respectively, where the errors represent the $1 \sigma$ dispersion on the mean. The values derived using the $\mathrm{F}$ and $\mathrm{K}$ template spectra are different because the intrinsic photospheric absorption line widths are smaller in the two $\mathrm{K}$ stars. It is surprising to find that the K3 Iab star in our sample has the same line widths as the $\mathrm{K} 1$ giant, suggesting the K3 star may be wrongly classified in terms of its luminosity. The velocity dispersions derived from cross-correlating the two K stars (HR 1529 and 6498) with the F8 bright giant are $8.8 \pm 0.6$ and $8.7 \pm 0.3 \mathrm{~km} \mathrm{~s}^{-1}$ respectively, and are entirely consistent with the differences in the cluster velocity dispersions found from the various template stars.

To choose the appropriate velocity dispersion value for M82-F, we need to determine which template star provides the best spectral/luminosity match and consider the effects of macroturbulent broadening in the atmospheres of cool evolved stars. In bright giants macroturbulence is the dominant factor in setting weaker stellar absorption line profile widths, and this depends primarily on luminosity class for cooler stars. As discussed above, the closest spectral match is to the two K stars. Inspection of the Starburst99 code (Leitherer et al. 1999) shows that K II stars dominate the UES wavelength region at an age of $60 \mathrm{Myr}$ (Paper I). We thus choose the F8 II template star since Gray \& Toner (1986) show that the macroturbulent broadening in F5-K4 luminosity class II bright giants is very similar with a mean value of $7.2 \pm 1.5 \mathrm{~km} \mathrm{~s}^{-1}$, while the K III stars have sharper lines, in agreement with our results.

We therefore adopt a velocity dispersion $\sigma$ of $13.4 \pm$ $0.7 \mathrm{~km} \mathrm{~s}^{-1}$ for M82-F. M82-F has a sufficiently large stellar velocity dispersion to be a proto-globular cluster.

\subsection{The Dynamical Mass}

The mass of M82-F can be obtained from the virial theorem since the average stellar crossing time of $\approx 4 \times 10^{5} \mathrm{yr}$ is much less than the cluster age, and thus the cluster should be near virial equilibrium. The virial mass $M$ is given by

$M=7.5 \frac{\sigma^{2} r_{\mathrm{m}}}{G}$

where it is assumed that the cluster is spherical with an isotropic velocity distribution such that $\sigma$ represents the one-dimensional rms velocity dispersion along the line-ofsight (Spitzer 1987). With the radius expressed in terms of the half-mass radius $r_{\mathrm{m}}$ (rather than the gravitational radius), the constant of proportionality is 7.5 (see e.g. the review of Gerhard 2000). Further assuming that the half-light radius derived from the WFPC2 image (Sect. 2.1) represents the projected half-mass radius and taking $r_{\mathrm{m}}=4 / 3 r_{\mathrm{h}}$ (Spitzer 1987), we derive a mass of $1.2 \pm 0.1 \times 10^{6} \mathrm{M}_{\odot}$, and a mass density within the half-mass radius of $6.4 \pm$ $0.9 \times 10^{3} \mathrm{M}_{\odot} \mathrm{pc}^{-3}$. M82-F is clearly a very massive young cluster that might become a globular cluster.

\subsection{The Luminosity-to-Mass Ratio}

One of the most important parameters to derive when considering whether M82-F will survive to become an old globular cluster is the luminosity-to-mass ratio $\left(L_{V} / M\right) \odot$ (see discussion in Sect. (1). This tells us if a cluster has sufficient mass in the form of lower luminosity, long-lived stars to avoid possible dynamical disruption. To determine this ratio we require the absolute magnitude and thus the reddening towards M82-F. O'Connell \& Mangano (1978) estimated $E(B-V) \approx 1.0$. In Paper I, we estimated a reddening of $\sim 1.5$ by comparing the theoretical flux distribution of a 60 Myr model cluster spectrum to the blue spectrum of M82F. We found that a standard Galactic extinction model did 
Table 1. Summary of derived parameters for M82-F

\begin{tabular}{ll}
\hline Parameter & Value \\
\hline Age & $60 \pm 20 \mathrm{Myr}$ \\
Half-light radius $r_{\mathrm{h}}$ & $2.8 \pm 0.3 \mathrm{pc}$ \\
$V$ & $16.12 \pm 0.10$ \\
$(B-V)$ & $1.22 \pm 0.10$ \\
$\left(V-I_{\mathrm{C}}\right)$ & $1.54 \pm 0.10$ \\
Radial velocity $V_{\mathrm{r}}$ & $+24.3 \pm 1.7 \mathrm{~km} \mathrm{~s}^{-1}$ \\
Velocity dispersion $\sigma$ & $13.4 \pm 0.7 \mathrm{~km} \mathrm{~s}^{-1}$ \\
Mass $M$ & $1.2 \pm 0.1 \times 10^{6} \mathrm{M}_{\odot}$ \\
Density $\rho$ & $6.4 \pm 0.9 \times 10^{3} \mathrm{M}_{\odot} \mathrm{pc}^{-3}$ \\
$E(B-V)$ & $0.9 \pm 0.1$ \\
$M_{V}\left(L_{V} / L_{\odot}\right.$ & $-14.5 \pm 0.3$ \\
$\log L_{V}$ & $7.73 \pm 0.12$ \\
$\left(L_{V} / M\right) \odot$ & $45 \pm 13$ \\
\hline
\end{tabular}

not correct the data in the near-UV. We now realise that this disparity is partially due to contamination from the close companion at the bluest wavelengths since this cluster was in the slit.

We are now able to obtain an accurate value for the reddening towards M82-F by comparing the WFPC2 $(B-V)$ and $(V-I)$ observed colours with the colours predicted for a 60 Myr old cluster. From the spectral synthesis models, Starburst99 (Leitherer et al. 1999) and PEGASE (Fioc \& Rocca-Volmerange 1997), we derive $E(B-V)=0.86$ and 0.82 using the observed $(V-I)$ colour given in Table 1, and correcting for the different photometric systems used in the two synthesis models. From the observed $(B-V)$ colour, we obtain $E(B-V)=1.11$ and thus adopt a reddening towards $\mathrm{M} 82-\mathrm{F}$ of $0.9 \pm 0.1$ where the error reflects the range in the reddening derived from the different photometric indices.

In Table 1, we list all the parameters derived for M82F. Using these values, we obtain $M_{V}=-14.5 \pm 0.3$, and a luminosity-to-mass ratio $\left(L_{V} / M\right)_{\odot}=45 \pm 13$. In Fig. 17, the predicted variation of $M_{V}$ with age is shown for a cluster of the same mass as M82-F at 60 Myr with a standard IMF where the slope is flatter below $0.5 \mathrm{M}_{\odot}$. We have adopted the parameterisation of Kroupa (2001): $\alpha=1.3$ for masses in the range $0.1 \leqslant M<0.5 M_{\odot}$ and $\alpha=2.3$ for $M \geqslant 0.5 \mathrm{M}_{\odot}$. We used the Starburst99 population synthesis code (Leitherer et al. 1999) with lower and upper mass limits equivalent to 0.1 and $100 \mathrm{M}_{\odot}$. Mass-loss due to stellar winds and supernovae are accounted for using the mass-loss rates of the Geneva models and assuming that stars more massive than $8 M_{\odot}$ explode as supernovae and leave $1.4 M_{\odot}$ remnants.

It is clear that M82-F is too bright for its age by $\approx 1.7 \mathrm{mag}$ (a factor of 5 ). A standard IMF would give an acceptable fit to the measured $\left(L_{V} / M\right)_{\odot}$ only if M82$\mathrm{F}$ was near the peak of its optical luminosity, with an age of $\sim 8$ Myr. We thus find that our derived parameters for M82-F strongly suggest an abnormal MF. We now review the likely sources of error and their impacts on our derived parameters for M82-F before discussing this result. The considerable discrepancy shown in Fig. 0 indicates that we are looking for large factors in the uncertainties.

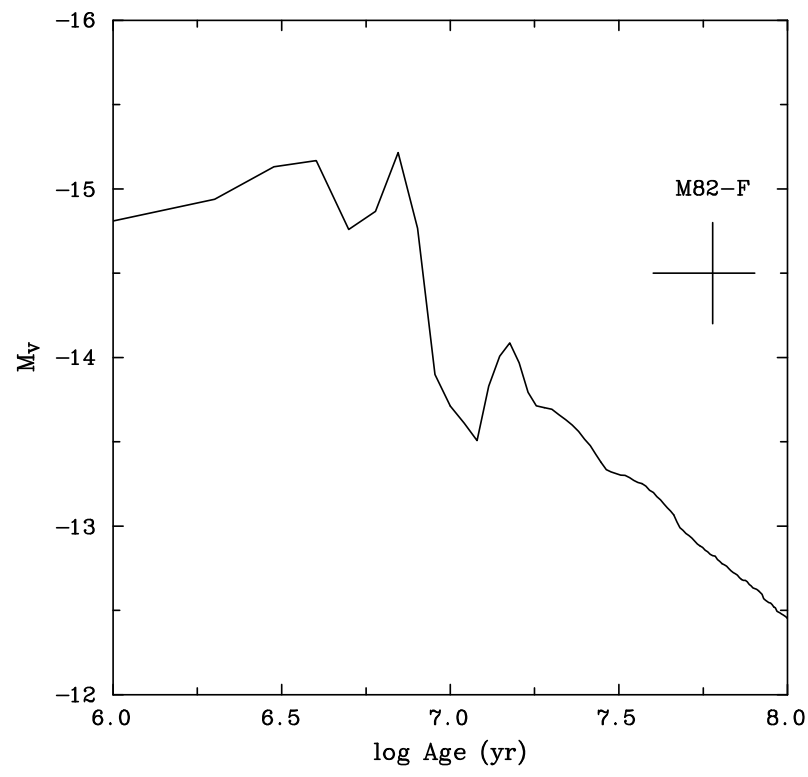

Figure 7. The absolute magnitude $M_{V}$ plotted as a function of age for a cluster of the same mass as M82-F at $60 \mathrm{Myr}$ for solar metallicity, a Kroupa (2001) IMF ( $\alpha=1.3$ for masses in the range $0.1 \leqslant M<0.5 M_{\odot}$ and $\alpha=2.3$ for $M \geqslant 0.5 \mathrm{M}_{\odot}$ ), lower and upper mass limits of 0.1 and $100 \mathrm{M}_{\odot}$, calculated using the Starburst99 code (Leitherer et al. 1999). The derived $M_{V}$ and age for M82-F are plotted. It is clearly too bright for its age by $\sim 1.7 \mathrm{mag}$, indicating that the IMF as shown is not appropriate for this cluster.

\section{UNCERTAINTIES}

\subsection{Age}

The age of M82-F is probably the most critical parameter since if it substantially younger than $60 \mathrm{Myr}$ old, then this will reduce the discrepancy we have found. We have therefore carried out a careful re-analysis of the age of M82-F as follows. In Paper I, we determined an age for M82-F by comparing the observed blue spectrum from 3510-5000 $\AA$ with model cluster spectra generated from the PEGASE spectral synthesis code (Fioc \& Rocca-Volmerange 1997). We used the Jacoby et al. (1984) spectral library, the 'Geneva' stellar evolutionary tracks and a Salpeter IMF. By matching the depth of the Balmer absorption lines and the Balmer jump, an age of $60 \pm 20 \mathrm{Myr}$ was determined. We noted that the spectral library of Jacoby et al. had lower resolution and often inferior signal-to-noise to the WHT+ISIS spectrum of cluster F. Since Paper I, a new synthetic library has become available for the $\mathrm{H}$ Balmer and He I absorption lines with a sampling of $0.3 \AA$ (González Delgado \& Leitherer 1999). Using this new dataset we have re-visited the age of M82-F by performing a detailed line profile comparison for the $\mathrm{H}$ Balmer series and the HeI lines.

We used the output of the evolutionary synthesis code Starburst99 (Leitherer et al. 1999) for an instantaneous burst and a Salpeter IMF with upper and lower mass cutoffs of 1 and $80 \mathrm{M}_{\odot}$ at solar metallicity. González Delgado, Leitherer \& Heckman (1999) find that the strengths and widths of the Balmer lines increase with age and that He I absorption reaches a maximum at $10-40 \mathrm{Myr}$ and is absent after 

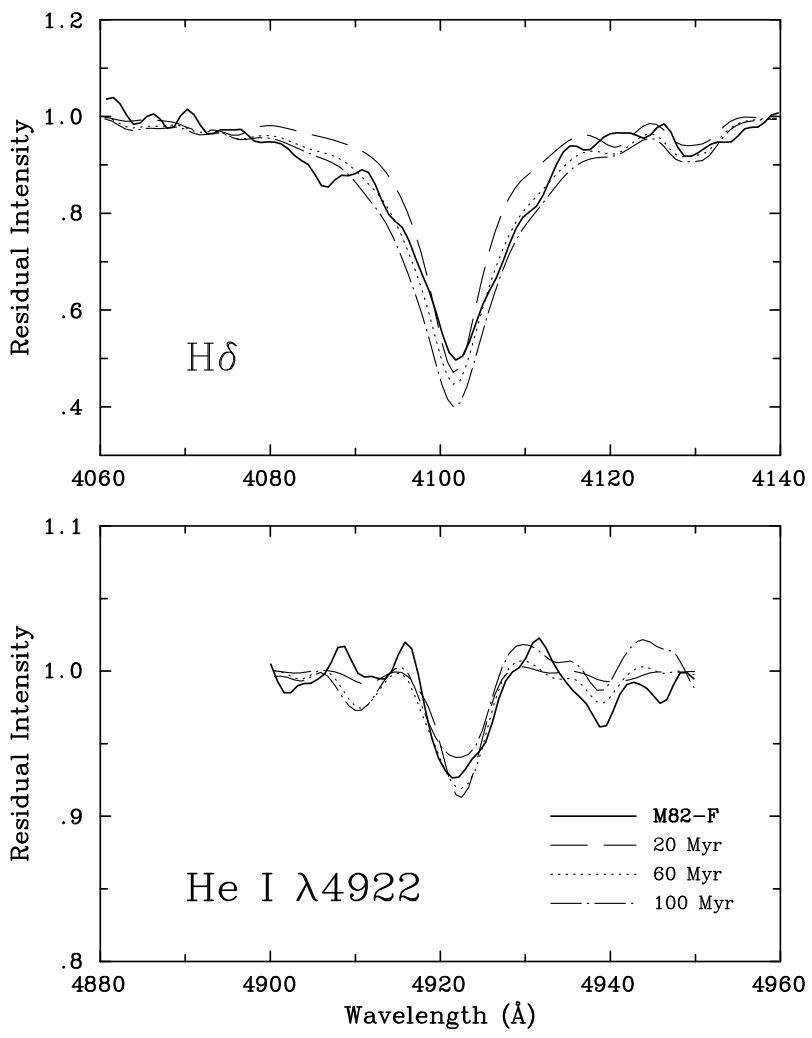

Figure 8. The observed $\mathrm{H} \delta$ and $\mathrm{He}$ I $\lambda 4922$ line profiles for M82F compared with synthetic profiles from the population synthesis models of González Delgado et al. (1999) for ages of 20, 60 and 100 Myr. The M82-F data were obtained with the WHT+ISIS and were presented in Paper I. The synthetic data have been binned to match the $1.6 \AA$ resolution of the M82-F data and all spectra have been smoothed using $\sigma=1.0 \AA$. An age of $60 \pm 20 \mathrm{Myr}$ is obtained from the best fits to the wings of the $\mathrm{H} \delta$ line profile (the core is affected by nebular emission) and the absorption depth of HeI $\lambda 4922$.

an age of $100 \mathrm{Myr}$. They also investigate the sensitivity of the Balmer lines to changes in the IMF parameters and find that a flatter IMF produces weaker Balmer features only for the first 4 Myr because of the greater proportion of $\mathrm{O}$ stars on the main sequence.

To compare observed and synthetic profiles, the M82-F spectrum was smoothed $(\sigma=1.0 \AA)$ and pseudo-continua were fitted to the $\mathrm{H} \beta-\mathrm{H} 13$ and HeI profiles using the windows defined in González Delgado \& Leitherer (1999). The synthetic spectra were treated in the same way and smoothed and binned to match the resolution of the M82-F spectrum. The observed and synthetic line profiles for $\mathrm{H} \delta$ and HeI $\lambda 4922$ are shown in Fig. 8 for ages of 20, 60 and 100 Myr. Since the cores of the lower members of the Balmer lines are affected by nebular emission, we use fits to the line profile wings as the main age discriminator. It can be seen that the $20 \mathrm{Myr} \mathrm{H}$ line profiles are too narrow and that He I $\lambda 4922$ is too strong. Conversely, at $100 \mathrm{Myr}$, the $\mathrm{H}$ lines are too broad and HeI is too weak. We thus confirm the age determined in Paper I of $60 \pm 20$ Myr.

Most importantly, M82-F cannot be as young as the age of $\sim 8$ Myr required for agreement between the predicted and observed $M_{V}$ shown in Fig. 6. At this age, RSGs will dominate but no molecular bands are detected (Paper I; cf. Bica, Santos \& Alloin 1990; Schiavon, Barbuy \& Bruzual $2000)$. The predicted $(V-I)$ colour at $8 \mathrm{Myr}$ gives a low $E(B-V)=0.4$ in contradiction to the $(B-V)$ colour, indicating that M82-F cannot have the colours of a starburst dominated by RSGs. If M82-F was as young as 4 Myr then we would expect to see Wolf-Rayet features in the spectra but these are clearly absent (Paper I).

\subsection{Luminosity and Radius}

M82-F is observed on a complicated background, as shown in Figs. 1 and 2. We have measured this 'background' relatively far from the cluster, and so could have made the cluster too bright. However, a 50 per cent increase in the background only decreases the observed $V$ by $0.1 \mathrm{mag}$. Furthermore, it seems equally likely that we could have underestimated the luminosity by not including light outside of our 0.7 arcsec radius aperture.

The measurements of the half-light radius are reasonably robust, and will only change slightly even if a moderate amount of luminosity has been missed. A key assumption here is that the half-light radius is the same as the projected half-mass radius. This cluster appears to be too young to have experienced significant dynamical mass segregation since the half-mass relaxation time (Spitzer \& Hart 1971) is $\approx 2 \mathrm{Gyr}$ giving a mass segregation time scale of $\approx 160 \mathrm{Myr}$ for stars with masses near the main sequence turn-off at the age of M82-F. Massive stars could, however, be centrally concentrated in M82-F due to processes at birth or associated with early evolution (see discussion in Sect. 7.2). Our procedure to correct saturated pixels could have further exacerbated this effect if it exists. If massive stars are extremely centrally concentrated in M82-F, we could have underestimated $r_{h}$ and therefore the mass.

\subsection{Extinction}

The range of possible interstellar obscurations remains a major source of uncertainty. The combination of spectrophotometry in Paper I and results presented here suggest that the colour excess is unlikely to be $E(B-V)<0.8$. Furthermore if we are not in the pure interstellar extinction case, then the absorption will be larger at a fixed colour excess (e.g. Calzetti, Kinney \& Storchi-Bergmann 1994). The best way to remove this as an issue would be to obtain high angular resolution infrared $K$-band images which would minimize the effects of interstellar obscuration. For the present it seems more likely that we have, if anything, underestimated the amount of visual obscuration towards M82-F.

We must also consider that we have used the predictions of stellar synthesis codes to provide the intrinsic colour. These were based on a Salpeter IMF with upper and lower mass cutoffs of 0.1 and $120 \mathrm{M}_{\odot}$. For a flatter IMF with $\alpha=1.5$, the predicted $(B-V)$ and $(V-I)$ colours differ by less than $0.1 \mathrm{mag}$ at an age of $60 \mathrm{Myr}$.

\subsection{Distance}

We have assumed that M82 and M81 are at the same distance and adopt the M81 distance of 3.6 Mpc (Freedman 
et al. 1994). A new distance estimate by Sakai \& Madore (1999) places M82 about $1 \sigma$ or $0.4 \mathrm{Mpc}$ further away than our adopted value. This will increase the luminosity to mass ratio by about 10 per cent.

\subsection{Velocity Dispersion}

We adopted the velocity dispersion value derived from the cross-correlation function of M82-F with an F8 II template star. If instead, we had chosen a velocity dispersion value given by one of the narrower-lined template stars, this would increase the dispersion by a maximum amount of 15 per cent, and the mass estimate by 30 per cent.

\subsection{Metallicity}

We have assumed that the metallicity of M82-F is solar. McLeod et al. (1993) summarise and discuss the metallicity of M82. They conclude that the present-day interstellar medium has solar or slightly greater than solar metallicity.

\subsection{Mass Model}

In Sect. 4.2 we followed the approach adopted by earlier studies in using the virial theorem applied at the half-mass radius to calculate the dynamical mass (e.g., Ho \& Filippenko 1996a,b). However, other dynamical models for star clusters yield different masses. For example, Dubath \& Grillmair (1997) derived masses for globular star clusters in M31 from integrated velocity dispersions using both King models and the virial theorem. They find King models yield masses that are $60-80 \%$ of those derived from the virial theorem applied to the same globular star clusters. That this effect is possible can be seen from the form of the Jeans equation (e.g., Binney \& Tremaine 1987), which also could allow our virial mass to be an underestimate in some cases.

We have also assumed that M82-F is spherical with an isotropic velocity distibution, but the real shape is elliptical (see Fig. 2). We can estimate the effect of an anisotropic velocity distribution on the dynamical mass by assuming that we are viewing the cluster along one long axis. In this case, we are likely to be overestimating the mass by about 30 per cent since we have overestimated the internal energy. We therefore conclude that our mass estimate could be uncertain by a factor of (up to) $\pm 50 \%$ if M $82-\mathrm{F}$ has a dynamical structure similar to that of globular star clusters.

In summary, we conclude that our errors appear to provide a reasonable assessment of the uncertainties as they are currently understood. There is no clear path that would lead to a mass increase by a factor of several. The largest remaining ambiguity is with interstellar obscuration, and this should be removed by future IR photometry.

\section{PARAMETERS OF SSCS NGC 1569A AND NGC 1705-1}

Stellar velocity dispersions have been measured by Ho \& Filippenko (1996a,b) for the young SSCs NGC 1569A and NGC 1705-1. Sternberg (1998) derived their luminosity-tomass ratios using revised parameters and found that NGC 1705-1 could have a top-heavy MF while NGC 1569A has
Table 2. Comparison of Properties of Super Star Clusters with Dynamical Mass Measurements

\begin{tabular}{llll}
\hline $\begin{array}{l}\text { Cluster/ } \\
\text { Parameter }\end{array}$ & NGC 1569A & NGC 1705-1 & M82-F \\
\hline & & & \\
Age (Myr) & $4-10^{a}$ & $10-20^{b}$ & $60 \pm 20$ \\
Metallicity $\left(\mathrm{Z}_{\odot}\right)$ & $0.20^{c}$ & $0.45^{d}$ & $1.0^{e}$ \\
$\mathrm{M}_{V}(\mathrm{mag})$ & $-14.0 \pm 0.4^{a}$ & $-13.8 \pm 0.4$ & $-14.5 \pm 0.3$ \\
$\mathrm{r}_{h}(\mathrm{pc})$ & $2.3 \pm 0.3^{a}$ & $1.6 \pm 0.4$ & $2.8 \pm 0.3$ \\
$\left.\sigma(\mathrm{km} \mathrm{s})^{-1}\right)$ & $15.7 \pm 1.5^{f}$ & $11.4 \pm 1.5^{g}$ & $13.4 \pm 0.7$ \\
$\mathrm{Mass}\left(\times 10^{6} \mathrm{M}_{\odot}\right)$ & $1.3 \pm 0.2$ & $0.48 \pm 0.12$ & $1.2 \pm 0.1$ \\
$\left(L_{V} / M\right)_{\odot}$ & $26 \pm 11$ & $60 \pm 24$ & $45 \pm 13$ \\
\hline
\end{tabular}

${ }^{a}$ Hunter et al. (2000); ${ }^{b}$ Heckmann \& Leitherer (1997); ${ }^{c}$ Kobulnicky \& Skillman (1997); ${ }^{d}$ Devost, Roy \& Drissen (1997); ${ }^{e}$ McLeod et al. (1993); ${ }^{f}$ Ho \& Filippenko (1996a); ${ }^{g}$ Ho \& Filippenko (1996b)

a steep MF with a slope of $\sim 2.5$. Since this study, the structural characteristics of the two SSCs have been further updated, and we have therefore re-derived their luminosities and masses for comparison with M82-F, as shown in Table 2. The two nearest SSCs, NGC 3603 in the Galaxy and R136a in the LMC, do not have velocity dispersion measurements and therefore cannot be compared directly with our results for M82-F.

\section{$6.1 \quad$ NGC $1569 \mathrm{~A}$}

The star clusters in the nearby starburst galaxy NGC 1569 have been studied most recently by Hunter et al. (2000) using new HST WFPC2 data. We will use their parameters, adopting a distance to NGC 1569 of $2.5 \mathrm{Mpc}$ (O'Connell et al. 1994). O'Connell et al. (1994) first noted that NGC 1569A has two peaks in its light distribution from WFPC images, suggesting it may consist of two sub-clusters A1 and A2. This was later verified by De Marchi et al. (1997) using WFPC2 data. González Delgado et al. (1997) suggested that the two sub-clusters have different ages of 3 and 9 Myr on the basis of Wolf-Rayet (WR) and red supergiant (RSG) features detected in ground-based spectroscopy.

Hunter et al. (2000) performed a careful analysis of the structure of NGC 1569A and find that the two components have similar colours, with A2 being fainter than A1 by 1.7 mag in the F555W filter. They suggest that there is no contradiction in the presence of both WR and RSG stars at the same age in a low metallicity environment, or alternatively that there may be a small age spread of several Myr in NGC 1569A. We therefore adopt an age for this cluster of 4-10 Myr, and assume that the velocity dispersion measurement of Ho \& Filippenko (1996a) represents the entire cluster. We derive a mass of $1.3 \pm 0.2 \times 10^{6} \mathrm{M}_{\odot}$ and $\left(L_{V} / M\right)_{\odot}=26 \pm 11$. The uncertainties were determined using the errors given in Table 2, where the error on $M_{V}$ is derived from the uncertainty in the reddening discussed by Hunter et al. (2000). 


\subsection{NGC 1705-1}

NGC 1705 is a nearby dwarf galaxy which is dominated by a single super star cluster, NGC 1705-1. The main observational uncertainties in previous mass derivations for NGC 1705-1 have been the half-light radius and the distance. As discussed in Sect. 2.1, measurements based on WFPC data have ranged from $0.9-3.4 \mathrm{pc}$ for a distance of $5.0 \pm 2.0 \mathrm{Mpc}$ (Meurer et al. 1995; O'Connell et al. 1994). Using archival WFPC2 images, we derive $r_{\mathrm{h}}=1.6 \pm 0.4 \mathrm{pc}$, using a new distance estimate of $5.3 \pm 0.8 \mathrm{Mpc}$ (Tosi et al. 2001, in prep.) With the velocity dispersion determined by Ho \& Filippenko (1996b), we derive a mass of $4.8 \pm 1.2 \times 10^{5} \mathrm{M}_{\odot}$ and $\left(L_{V} / M\right)_{\odot}=60 \pm 24$, with $V=14.9 \pm 0.1$ (Sect. 2.1).

\section{DISCUSSION}

\subsection{The Top-Heavy MF}

In Table 2, we list the parameters of NGC 1569A, NGC 1705-1 and M82-F. The absolute magnitude and mass of M82-F are similar to NGC 1569 A but M82-F is a factor of 3-6 times older and thus it is exceptionally bright. Sternberg (1998) derived $\left(L_{V} / M\right)_{\odot}=29$ for NGC 1569A and found by comparison with population synthesis models that this cluster has a steep MF $(\alpha \sim 2.5)$ and will probably evolve to a globular cluster-type object. Conversely, he derived $\left(L_{V} / M\right)_{\odot}=126$ for NGC 1705-1 and found that the $\mathrm{MF}$ is either flat $(\alpha<2)$ or truncated between $1-3 \mathrm{M}_{\odot}$, implying that this cluster will not survive. While the $\left(L_{V} / M\right) \odot$ we derive for NGC 1569A is identical within the errors to the Sternberg value, the revised value for NGC 1705-1 is a factor of $\sim 2$ lower.

To investigate the form of the mass function for M82-F and the two other SSCs, we have calculated $\left(L_{V} / M\right) \odot$ as a function of age for various IMF parameterisations with the Starburst99 code. Spectral synthesis codes usually make the unrealistic assumption that the mass-loss due to stellar evolution remains in the cluster. In calculating $\left(L_{V} / M\right)_{\odot}$, we have therefore allowed for a decreasing cluster mass by subtracting the stellar ejecta at each time step.

We adopt the parameterisation of Kroupa (2001) to represent our IMF model: $\alpha=1.3$ for masses in the range $0.1 \leqslant M<0.5 M_{\odot}$ and $\alpha=2.3$ for $M \geqslant 0.5 \mathrm{M}_{\odot}$ with an upper mass limit $m_{\mathrm{u}}=100 \mathrm{M}_{\odot}$, and lower mass limits in the range $m_{1}=0.1-3 \mathrm{M}_{\odot}$. These models are shown in Fig. 9 . It is clear that there is a fundamental mismatch by a factor of $\sim 5$ between the derived $\left(L_{V} / M\right)_{\odot}$ for M82-F and that predicted by a 'simple stellar population' (SSP) model with a standard Kroupa (2001) IMF with $m_{1}=0.1 \mathrm{M}_{\odot}$.

Both NGC 1569A and NGC 1705-1 have metallicities below solar (Table 2). We have investigated the effect of a lower metallicity on $\left(L_{V} / M\right) \odot$ by running the Starburst99 code for $Z=0.2$ and $0.4 Z_{\odot}$. We find that the difference in $\left(L_{V} / M\right)_{\odot}$ is well within the observational errors because at lower metallicities and young ages, the luminosity is increased but the mass loss rate is lower. Sternberg (1998) finds that NGC 1569A has a steep MF with $\alpha \sim 2.5$, extending to $0.1 \mathrm{M}_{\odot}$. This is confirmed in Fig. 9 where we show $\left(L_{V} / M\right) \odot$ as a function of time for the Sternberg (1998) IMF parameters. For NGC 1705-1, Sternberg (1998) finds that the MF is either flat or truncated. Our revised $\left(L_{V} / M\right) \odot$ for

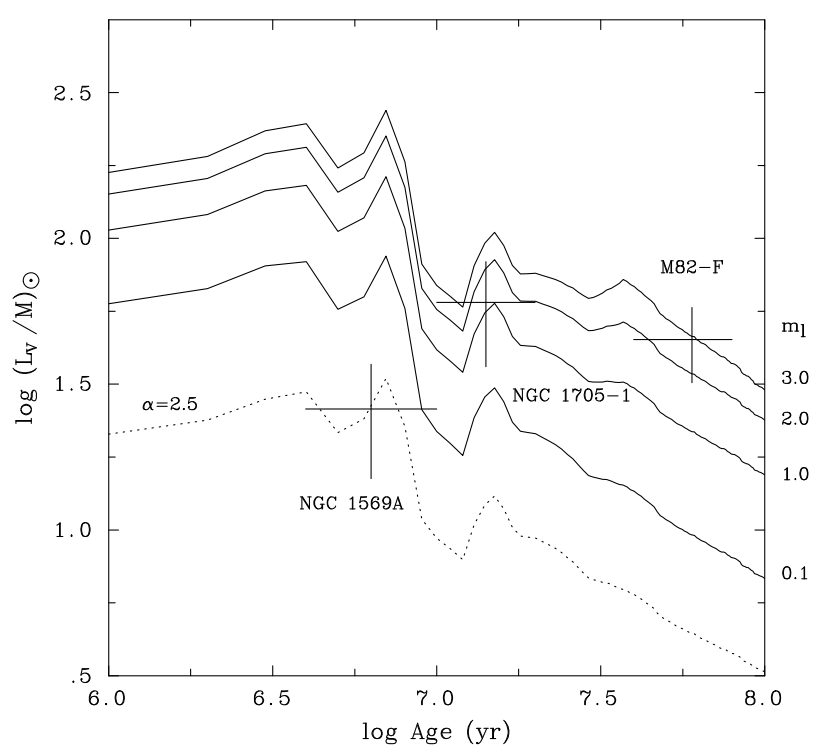

Figure 9. $\left(L_{V} / M\right)_{\odot}$ plotted as a function of age using the Starburst99 code (Leitherer et al. 1999) taking into account massloss due to stellar evolution. The solid curves shown are for solar metallicity, an IMF parameterised by $\alpha=1.3$ for $0.1 \leqslant M<$ $0.5 M_{\odot}$ and $\alpha=2.3$ for $M \geqslant 0.5 \mathrm{M}_{\odot}$, an upper mass cut-off of $100 \mathrm{M}_{\odot}$, and lower mass cut-offs of $0.1,1.0,2.0$ and $3.0 \mathrm{M}_{\odot}$. The derived $\left(L_{V} / M\right)_{\odot}$ and age values for M82-F, NGC 1569A and NGC 1705-1 are plotted. The dotted curve is for solar metallicity, a single slope $\alpha=2.5$, and lower and upper mass cut-offs of 0.1 and $100 \mathrm{M}_{\odot}$.

this cluster is lower by a factor of 2 but it still lies well above the IMF curve extending down to $m_{1}=0.1 \mathrm{M}_{\odot}$ (Fig. 9), indicating that NGC $1705-1$ probably has a truncated MF with $m_{l} \approx 1-3 \mathrm{M}_{\odot}$.

The high visual luminosity-to-mass ratio that we have derived for M82-F indicates that this SSC has an abnormal MF with a minor fraction of its stellar mass concentrated in long-lived, low mass stars. To explore this possibility, we change the mass distribution by raising the lower cut-off mass in the SSP models until we fit the observed $\left(L_{V} / M\right)_{\odot}$ ratio for M82-F. As shown in Fig. 9, we need $m_{1}=2-3 \mathrm{M}_{\odot}$. A similar lower mass range is indicated for a flatter IMF with a slope of 2.0. This exercise then implies a 'top-heavy' MF where most of the stellar mass is in stars with $\mathrm{M}>2 \mathrm{M}_{\odot}$ in the tightly gravitationally bound regions of the cluster.

This conclusion merits careful examination; previous claims for highly abnormal MFs often have proven incorrect. Scalo (1998) summarized the evidence for variations in the IMF. A key point from Scalo's review is that most documented IMF variations seem to occur within individual starforming regions. On large scales, the present-day IMF seems to be remarkably constant (e.g. Kroupa 2001), and this trend appears to extend to the MFs of the nearby SSCs NGC 3603 and R136a (see discussion in Sect. 11). Thus it is not clear whether M82-F represents a high- and intermediate-mass star-enriched core of a larger star-forming complex, within which the overall MF is relatively normal (see Sect. 7.2).

Some super star clusters appear to have formed as part of a more extended region, e.g. knot $\mathrm{S}$ in the 'antennae' interacting galaxies (Whitmore et al. 1999) or the 'nucleus' 
of the peculiar irregular galaxy NGC 4449 (Gelatt, Hunter \& Gallagher 2001; Böker et al. 2001), while in other cases, such as NGC 1569A, the SSC is well separated from its surroundings (Hunter et al. 2000), and has a normal MF (Fig. 9; Sternberg 1998).

The Galactic centre Arches and Quintuplet star clusters provide the most compelling local examples of MF anomalies. Figer et al. (1999) find both of these compact, moderate mass $\left(\sim 10^{4} \mathrm{M}_{\odot}\right)$ systems have peculiarly flat MFs for $\mathrm{M}>10 \mathrm{M}_{\odot}$. They attribute this behaviour to the strong tidal field near the Galactic centre that could inhibit the formation of lower mass stars.

Or is M82-F an example of an abnormal mode of star formation of the type suggested from Kroupa's (2001) anaylsis of Galactic globular clusters? With respect to this point, we can see from Fig. 9 that an SSP model with a single IMF is unlikely to fit all three SSCs with mass determinations. A larger sample of SSCs with mass and size determinations is needed to resolve properly this issue. Until we do so it is premature to generalize our result to the overall pattern of star formation in M82.

\subsection{Formation and Mass Segregation}

Tightly bound star clusters are now widely recognized to be products of locally efficient star formation; typically half or more of the gas in the initial cloud's densest region must be converted into stars to yield these types of objects (e.g. Goodwin 1997). M82-F must have originated in a molecular cloud core with a density of $n \sim 10^{5} \mathrm{~cm}^{-3}$ and a very high pressure (Elmegreen \& Efremov 1997). The discussion of these points by Ho and Filippenko (1996b) on the connection between massive SSCs and ultra-dense molecular clouds in M82 is borne out by our observations.

The high peculiar velocity of M82-F then is interesting. At present neither dense molecular gas nor H II emission are found at the cluster's velocity, blueshifted by almost $170 \mathrm{~km} \mathrm{~s}^{-1}$ with respect to the centroid of M82. The cluster evidently has had time to completely separate itself from any natal gas. This is consistent with our finding that the cluster is old enough to have made more than one revolution about the centre of M82; it is now well-removed from its birthplace and faces an uncertain future.

As discussed in Sect. 5.2, M82-F is too young to have experienced significant dynamical mass segregation. Some star clusters, however, seem to have been born with their massive stars centrally concentrated. The Orion nebula cluster sets an example of this phenomenon; massive stars are centrally located but the cluster is far too young for two-body relaxation to have played a role (Hillenbrand \& Hartmann 1998). Another example, NGC 2098 in the LMC, has a similar age to M82-F, and shows some signs of mass segregation (Kontizas et al. 1998). Fischer et al. (1998) reach a similar conclusion for the $100 \mathrm{Myr}$ old LMC star cluster NGC 2157. Thus, if mass-segregation is playing a significant role in M82-F, it must have been present at birth.

M82-F should therefore have an extended halo of low mass stars if primordial mass segregation has occurred. To test for this possibility, we take an extreme model where the velocity dispersion is the same and assume that low mass stars affect the mass and not the luminosity. The half-light radius has then to increase by a factor of 5 to $14 \mathrm{pc}$ for
$\left(L_{V} / M\right)_{\odot}$ to agree with a Kroupa-type IMF. For comparison, in Fig. 2, clusters $\mathrm{F}$ and $\mathrm{L}$ are $\approx 70 \mathrm{pc}$ apart. This increase is well beyond the radius where we can detect M82-F, and while not impossible, there is no evidence at present to support such an extended halo. The other alternative is that M82-F has had its halo of low mass stars removed by a process such as tidal stripping. This mechanism has been proposed to explain the peculiar mass functions of the Galactic centre clusters (Gerhard 2001). While M82-F does have a peculiar orbit, it seems unlikely that it could have been born in a location with large tidal stresses because such an environment is not obviously present in M82. The data for the two younger SSCs NGC 1569A and 1705-1 are inconclusive. The former cluster appears to have a normal MF while the latter has a truncated MF yet both clusters reside in the inner parts of their galaxies.

We conclude that while mass segregation at birth may have occurred in M82-F, the current observational evidence favours the view that the IMF of M82-F was abnormal in being deficient in low mass stars. Future spatially-resolved spectroscopy of M82-F is needed to investigate the significance of mass segregation.

\subsection{Long Term Survival?}

Predicting the lifetime of a star cluster is difficult. The simplest model assumes a cluster is in virial equilibrium and therefore must expand in response to any mass loss. In the case of M82-F this will be dominated by effects due to stellar evolution. At the age of M82-F, the SSP models with lower mass cut-offs of 2 and $3 \mathrm{M}_{\odot}$, show that 49 and 60 per cent of the original mass has been ejected. Therefore, the cluster should soon have too little mass to remain bound with a constant stellar velocity dispersion; i.e. it will reach positive total energy. How long the cluster can endure depends on the actual form of the IMF, but since we expect that less than half of the original stellar mass resides in stars with $\mathrm{M}<2 \mathrm{M}_{\odot}$, the cluster should begin to dissolve in the next 1-2 Gyr as stars in this mass range complete their evolution. We therefore adopt a probable lifetime for the SSC M82-F in its present massive form of $\leqslant 2$ Gyr.

Of course star clusters are subject to a variety of internal and external evolutionary influences beyond those imposed by stellar evolution and the IMF, including tidal radius limits, stellar mass segregation, and the nature of the stellar velocity distribution function (see Chernoff \& Shapiro 1987). The general impact of these additional influences is to promote cluster expansion and thereby reduce the cluster lifetime, since expansion causes increased vulnerability of stars to tidally-induced mass loss (Spitzer 1987; Chernoff \& Weinberg 1990).

A cluster's long term prospects further depend on its orbit, and the importance of dynamical heating of its stars by processes such as disc shocking (Gnedin \& Ostriker 1997). Models of dense star clusters therefore generally require a steep IMF extending to masses well below that of the Sun to insure cluster survival over cosmic time scales. For clusters with lower mass limits of $\sim 0.1 \mathrm{M}_{\odot}$, IMF slopes of $\alpha \geqslant 2.3$ 2.5 are preferred for durable clusters in the Milky Way, and clusters with $\alpha \leqslant 1.5$ will disrupt even when the tidal field is weak, as in the Magellanic Clouds (e.g. Chernoff \& Weinberg 1990). Thus again, we are left with the question of whether 
the ancient globular clusters, and especially those near the dense centres of giant galaxies, were formed by some process that was preferred at early epochs (see Zhang \& Fall 1999)? Alternatively, it may simply be that the survivors were picked out from what was initially a varied population of SSCs (e.g. Gnedin \& Ostriker 1997).

The large radial velocity of $\mathrm{M} 82-\mathrm{F}$ with respect to its host galaxy implies that M82-F cannot be on a simple circular orbit within the disc of M82. It may be subject to some degree of disc shocking as well as a moderate tidal radius constraint of about $35 \mathrm{pc}$ produced by M82. This is sufficiently large that tides should not have an immediate impact on the structure of M82-F. However, if the cluster begins to expand due to mass loss, as expected within the next 2 Gyr, then tides will help to disperse the escaping stars.

\section{CONCLUSIONS}

We measure a stellar radial velocity dispersion of $13.4 \pm 0.7 \mathrm{~km} \mathrm{~s}^{-1}$ for the super star cluster M82-F. This result in combination with a new determination of the projected half-light radius of $r_{\mathrm{h}}=2.8 \pm 0.3 \mathrm{pc}$ yields a dynamical mass estimate of $1.2 \pm 0.1 \times 10^{6} \mathrm{M}_{\odot}$ for $\mathrm{M} 82-\mathrm{F}$. WFPC2 archival observations together with a revised estimate for the minimum amount of interstellar obscuration give $M_{V}=-14.5 \pm 0.3$ or $L_{V}=5.4 \pm 1.4 \times 10^{7} \mathrm{~L}_{\odot}$. The luminosity-to-mass ratio is then $\left(L_{V} / M\right) \odot=45 \pm 13$. These parameters show that M82-F has the luminosity and mass to qualify as a young globular star cluster.

Unlike most optically luminous SSCs, M82-F is middleaged; we reconfirm our earlier age estimate of $60 \pm 20 \mathrm{Myr}$ from Paper I based on fits to the $\mathrm{H} \delta$ and He I $\lambda 4922$ absorption line profiles. M82-F is a factor of 5 times too luminous for a star cluster of its age with a standard stellar mass function extending to $0.1 M_{\odot}$. Our data require that M82-F has a deficiency of low mass stars, at least within the optically bright component of the cluster.

M82-F appears to be unique for an object of its age in displaying the enhanced $\left(L_{V} / M\right) \odot$ predicted for top-heavy IMFs that are deficient in low mass stars. While this type of behaviour is seen in Galactic centre clusters for $\mathrm{M}>10 \mathrm{M}_{\odot}$, it is not yet known if this behaviour applies to lower stellar masses within these star clusters, or if such objects should be expected to exist outside of the very dense centres of giant galaxies. Our models suggest that the lower mass limit for $\mathrm{M} 82-\mathrm{F}$ is $2-3 \mathrm{M}_{\odot}$ for an IMF slope of 2.3 , and much of the mass is then near the lower stellar mass cutoff.

We have considered obvious sources of error but have found none that would substantially change our conclusions. In particular, we have investigated the question of mass segregation and find no current observational evidence to support this possibility. While modern forms of the IMF which have fewer low mass stars than a Salpeter IMF provide better agreement with our observed $\left(L_{V} / M\right) \odot$ ratio, they do not help with the dynamical problem of having sufficient low mass stars to hold the cluster together over cosmic time spans. In this case, the M82-F cluster is steadily losing mass due to mass lost by evolving stars, and should dissolve within the next 1-2 Gyr. If the cluster is limited to what we see, its high mass and density are not sufficient to assure its survival without sufficient mass in the form of long-lived, low mass stars. M82-F cannot then be considered as a proto-globular cluster.

The history of evidence for substantial variations in the IMFs of star clusters is chequered. For example, Elson, Fall \& Freeman (1989) suggested that intermediate mass stars have a flat IMF in some LMC star clusters, a view that gained support from an observed range in cluster core radii consistent with expansion due to mass loss in the presence of a flat IMF (Elson et al. 1989). However, in one of her last papers, Elson et al. (1999) present deep STIS photometry of two LMC star clusters that show rather normal MFs in both cases, even though one cluster has a compact core and the other a large core of the type previously associated with a flat IMF. In his review of IMFs, Scalo (1998) suggests that significant IMF variations may occur between star clusters, but finds no signs of IMF variations at the level we require in M82-F. Similarly, as we discussed earlier in Sect. 17, neither of the nearest SSCs, NGC 3603 and R136, have abnormal intermediate mass stellar MFs.

Further afield we re-examined properties of NGC 1569A from new structural information published by Hunter et al. (2000) and in NGC 1705-1 from size and photometric measurements taken from WFPC2 archival exposures for a new distance estimate (Tosi et al. 2001, in prep.). We confirm the features discussed by Sternberg (1998) for NGC 1569A which is well fit by SSP models with a slightly steeper IMF with a single slope of 2.5. The new data for NGC 1705-1 also confirm the results of Sternberg (1998) that this cluster has a top-heavy MF; for a $\alpha=2.3$ slope, we find that the $\mathrm{MF}$ is truncated near $2 \mathrm{M}_{\odot}$.

An indirect factor in favour of a possible top-heavy MF in M82-F is the long held suspicion that this type of situation might generally prevail in M82. Bernlöhr (1992) and Rieke et al. (1993) suggested a deficit of low mass stars from fits to the spectral energy distribution. Unfortunately, the luminosity of M82 depends critically on assumptions concerning the degree of interstellar obscuration. Satyapal et al. (1997) find that these earlier studies over-corrected for interstellar obscuration, leading to an artificially high stellar power output and the need for a top-heavy MF. Doane and Mathews (1993) took a different approach. In modelling the power requirements for the superwind, they needed a high supernova rate that could be naturally explained by a topheavy IMF. Again more modern approaches suggest that a normal IMF might be sufficient (Strickland \& Stevens 2000).

From what we presently know, the over-luminous cluster M82-F is an anomaly even within the anomalous M82 starburst. The only comparable cases may be those seen in NGC 1275 SSCs by Brodie et al. (1998), who presented spectroscopic evidence for top-heavy MFs. Yet theory suggests that if IMF variations occur, they are most likley to appear in dense star clusters, where mergers could take place and increase the mass where the stellar IMF breaks in slope (Larson 1999). The SSC M82-F then may be the long sought example of an evolved system with a truly peculiar MF (see Kroupa 2001), and its properties and environment therefore merit further careful investigation. 


\section{ACKNOWLEDGMENTS}

We thank Monica Tosi for providing the distance to NGC 1705-1 in advance of publication, and Richard Norris for his generous help with the Starburst99 code. We also thank John Mathis for his comments on this paper. Both authors thank the organisers of the Massive Stellar Clusters workshop held at the Observatory of Strasbourg in 1999 for a very informative and stimulating meeting where many of the ideas presented here were discussed. JSG similarly expresses his appreciation to participate in the Modes of Star Formation workshop that took place at the Max Planck Institut für Astronomie in Heidelberg during October 2000. JSG thanks the Vilas Trustees for a Vilas Associate through the University of Wisconsin-Madison Graduate School. Key support was provided by the U.S. National Aeronautics and Space Administration and Space Telescope Science Institute for studies of massive star clusters through research funds associated with Hubble Space Telescope observing programs, most recently as part of General Observer Program 7446. LJS thanks the Department of Astronomy at the University of Wisconsin-Madison for their warm hospitality and financial support during the writing of this paper. The William Herschel Telescope is operated on the island of La Palma by the Royal Greenwich Observatory in the Spanish Observatorio del Roque de los Muchachos of the Instituto de Astrofísica de Canarias. The WIYN Observatory is a joint facility of the University of Wisconsin-Madison, Indiana University, Yale University and the National Optical Astronomy Observatories.

\section{REFERENCES}

Achtermann J. M., Lacy J. H. 1995, ApJ, 439, 163 Ashman K.M., Conti A., Zepf S.E., 1995, AJ, 119, 1164

Bica E., Santos J.F.C., Alloin D.,1990, Revista Mexicana de Astronomia y Astrofisica, 21, 202

Bernlöhr K., 1992, A\&A, 263, 54

Binney J., Tremaine S., 1987, Galactic Dynamics, Princeton University Press, Princeton

Böker T., van der Marel R. P., Mazzuca L., Rix H.-W., Rudnick G., Ho L. C., Shields J. C., 2001, AJ, 121, 1473

Brodie J. P., Schroder L. L., Huchra J. P., Phillips A. C., KisslerPatig M., Forbes D. A. 1998, AJ, 116, 691

Calzetti D., Kinney A. L., Storchi-Bergmann T. 1994, ApJ, 429, 582

Chernoff, D. F., Shapiro, S. L. 1987, ApJ, 322, 113

Chernoff D.F., Weinberg M.D., 1990, ApJ, 351, 121

De Marchi G., Clampin M., Greggio L., Leitherer C., Nota A., Tosi M., 1997, ApJ, 479, L27

Devost D., Roy J.-R., Drissen L., 1997, ApJ, 482, 765

Doane J.S., Mathews W.G., 1993, ApJ, 419, 573

Dubath, P. \& Grillmair, C. J. 1997, A\&A, 321, 379

Eisenhauer F. et al 1998, ApJ, 498, 278

Elmegreen B., Efremov Y., 1997, ApJ, 480, 235

Elson R. A., Fall S. M., Freeman K. C. 1987, ApJ, 323, 54

Elson R. A., Fall S. M., Freeman K. C. 1989, ApJ, 336, 734

Elson R. A., Freeman K. C., Lauer T. R. 1989, ApJ, 347, L69

Elson R., Tanvir N., Gilmore G., Johnson R. A., Beaulieu, S. 1999, in Chu Y.-H., Suntzeff N. B., Hesser J. E., Bohlender D. A., eds., IAU Symp. No. 190. New Views of the Magellanic Clouds, Astron. Soc. Pac., San Francisco p. 417

Figer D. F., Kim S. S., Morris M., Serabyn E., Rich R. M., McLean I. S., 1999, ApJ, 525, 750
Fioc M., Rocca-Volmerange B., 1997, A\&A, 326, 950

Fischer P., Pryor C., Murray S., Mateo M., Richtler T., 1998, AJ, 115,592

Freedman W. et al., 1994, ApJ, 427, 628

Fritze - v. Alvensleben U., 1998, A\&A, 336, 83

Fritze - v. Alvensleben U., 1999, A\&A, 342, L25

Gallagher J.S., III, Smith L.J., 1999, MNRAS, 304, 540

Gelatt A. E., Hunter D. A., Gallagher J. S., 2001, PASP, 113, 142

Gerhard O., 2000, in Lançon A., Boily C.M., eds., ASP Conf. Ser. Vol. 211, Massive Stellar Clusters. Astron. Soc. Pac., San Francisco p. 12

Gerhard O., 2001, ApJL, 546, L39

Gnedin O. Y., Ostriker J. P. 1997, ApJ, 474, 223

González Delgado R.M., Leitherer C., 1999, ApJS, 125, 479

González Delgado R.M., Leitherer C., Heckman T.M., 1999, ApJS, 125, 489

González Delgado R.M., Leitherer C., Heckman T.M., Cerviño M., 1997, ApJ, 483, 705

Goodwin S. P. 1997, MNRAS, 286, 669

Götz M., McKeith C. D., Downes D., Greve A. 1990, A\&A, 240, 52

Gray D.F., Toner C.G., 1986, ApJ, 310, 277

de Grijs R., O'Connell R. W., Becker G. D., Chevalier R. A., Gallagher J. S., III, 2000, AJ, 119, 681

de Grijs R., O'Connell R. W., Gallagher J. S., III, 2001, AJ, 121, 768

Harris W. E. 1991, ARA\&A, 29, 543

Heckman T.M., Leitherer C., 1997, AJ, 114, 69

Hillenbrand L. A., Hartmann L. W., 1998, ApJ, 492, 540

Ho L.C., Filippenko A.V., 1996a, ApJ, 466, L83

Ho L.C., Filippenko A.V., 1996b, ApJ, 472, 600

Holtzman J.A., Burrows C.J., Casertano S., Hester J.J., Trauger J.T., Watson A.M., Worthey G., 1995, PASP, 107, 1065

Hunter D.A., O'Connell R.W., Gallagher J.S., Smecker-Hane Y.T., 2000, AJ, 120, 2383

Jacoby G.H., Hunter D.A., Christian C.A., 1984, ApJS, 56, 257

Kim S.S., Morris M., Lee H.M., 1999, ApJ, 525, 228

Kobulnicky H. A., Skillman E. D., 1997, ApJ, 489, 636

Kontizas M., Hatzidimitriou D., Bellas-Velidis I., Gouliermis D., Kontizas E., Cannon R. D., 1998, A\&A, 336, 503

Kroupa P. 2001, MNRAS, 322, 231

Larson R.B., 1985, MNRAS, 214, 379

Larson R. B. 1999, in T. Nakamoto, ed., Star Formation 1999, Nobeyama Radio Observatory, p.336-340,

Leitherer C. et al., 1999, ApJS, 123, 3

Lester D. F., Carr J. S., Joy M., Gaffney N. 1990, ApJ, 352, 544

Massey P., Hunter D. A. 1998, ApJ, 493, 180

McCleod K.K., Rieke G.H., Rieke M.J., Kelly D.M., 1993, ApJ, 412,111

Meurer G.R., Heckman T.M., Leitherer C., Kinney A., Robert C., Garnett D.R., 1995, AJ, 110, 2665

O'Connell R.W., Mangano J.J., 1978, ApJ, 221, 62

O'Connell R.W., Gallagher J.S., III, Hunter D.A., 1994, ApJ, 433, 65

O'Connell R.W., Gallagher J.S., III, Hunter D.A., Colley W.N. 1995, ApJ, 446, L1

Phillips A.C., Guzman R., Gallego J., Koo D.C., Lowenthal J.D., Vogt N.P., Faber S.M., Illingworth, G.D., 1997, ApJ, 489, 543

Rieke G.H., Lebofsky M.J., Thompson R.I., Low F.J., Tokunaga A.T., 1980, ApJ, 238, 24

Rieke G.H., Loken K., Rieke M.J., Tamblyn P., 1993, ApJ, 412, 99

Saitō M., Sasaki M., Kaneko N., Nishimura M., Toyama K., 1984, PASJ, 36, 305

Sakai S., Madore B.F., 1999, ApJ, 526, 599

Satyapal S. et al., 1995, ApJ, 448, 611

Satyapal S., Watson D.M., Pipher J.L., Forrest W.J., Greenhouse 
M.A., Smith H.A., Fischer J., Woodward C.E., 1997, ApJ, 483,148

Scalo J. 1998, in Gilmore G., Howell, D., eds., in ASP Conf. Ser. Vol. 142. The Stellar Initial Mass Function, Astron. Soc. Pac., San Francisco p. 201

Schiavon R.P., Barbuy B., Bruzual A.G., 2000, ApJ, 532, 453

Schweizer F., Seitzer P. 1993, ApJ, 417, L29

Seaquist E.R., Carlstrom J.E., Bryant P.M., Bell M.B., 1996, ApJ, 465,691

Shen J., Lo K. Y. 1995, ApJ, 445, L99

Shortridge K., Meyerdierks H., Currie M., Clayton M., 1997, Starlink User Note 86.13, Rutherford Appleton Laboratory

Sirianni M., Nota A., Leitherer C., De Marchi G., Clampin, M. 2000, ApJ, 533, 203

Sofue Y., Reuter H.-P., Krause M., Wielebinski R., Nakai N. 1992, ApJ, 395, 126

Spitzer L., Jr. 1987, Dynamical Evolution of Globular Clusters, Princeton Univ. Press, Princeton

Spitzer L., Jr., Hart M.H. 1971, ApJ, 164, 399

Sternberg A., 1998, ApJ, 506, 721

Strickland D. K., Stevens I. R. 2000, MNRAS, 314, 511

Takahashi K., Portegies Zwart S.F., 2000, ApJ, 535, 759

Telesco C. M. 1988, ARA\&A, 26, 343

Whitmore B.C., Schweizer F., Leitherer C., Borne K., Robert C., 1993, AJ, 106, 1354

Whitmore B.C., Zhang Q., Leitherer C., Fall S.M., Schweizer F., Miller B.W., 1999, AJ, 118, 1551

Yun M. S., Ho P. T. P., Lo K. Y. 1993, ApJ, 411, L17

Zhang Q., Fall S. M. 1999, ApJ, 527, L81

This paper has been typeset from a $\mathrm{T}_{\mathrm{E}} \mathrm{X} / \mathrm{I}_{\mathrm{E}} \mathrm{T} \mathrm{X}$ file prepared by the author. 